\title{
In vivo comparison of the biodistribution and toxicity of InP/ZnS quantum dots with different surface functional groups
}

\section{Li Li ( $\sim$ lily140222@163.com )}

Shenzhen University https://orcid.org/0000-0002-6789-2328

\section{Yajing Chen}

Shenzhen University

\section{Gaixia Xu}

Shenzhen University

Dongmeng Liu

Shenzhen University

\section{Zhiwen Yang}

Shenzhen University

\section{Tingting Chen}

Shenzhen University

Xiaomei Wang

Shenzhen University

Wenxiao Jiang

Shenzhen University

\section{Dahui Xue}

Shenzhen University

\section{Guimiao Lin}

Shenzhen University

\section{Research}

Keywords: InP/ZnS quantum dots, surface chemistry, in vivo, biodistribution, nanotoxicology

Posted Date: December 10th, 2019

DOI: https://doi.org/10.21203/rs.2.18407/v1

License: (c) (1) This work is licensed under a Creative Commons Attribution 4.0 International License.

Read Full License 
In vivo comparison of the biodistribution and toxicity of $\mathrm{InP} / \mathrm{ZnS}$ quantum dots with different surface functional groups

$\mathrm{Li} \mathrm{Li}^{1,2}$, Yajing $\mathrm{Chen}^{1}$, Gaixia $\mathrm{Xu}^{2,3}$, Dongmeng Liu ${ }^{1}$, Zhiwen Yang ${ }^{1}$, Tingting Chen $^{1}$, Xiaomei Wang ${ }^{1}$, Wenxiao Jiang ${ }^{1}$, Dahui Xue ${ }^{1}$, Guimiao Lin ${ }^{1 *}$

1 Base for International Science and Technology Cooperation: Carson Cancer Stem Cell Vaccines R\&D Center, Shenzhen Key Lab of Synthetic Biology, Department of Physiology, School of Basic Medical Sciences, Shenzhen University, Shenzhen 518055, China

2 Key Laboratory of Optoelectronics Devices and Systems of Ministry of Education/Guangdong Province, College of Optoelectronic Engineering, Shenzhen University, Shenzhen 518060, China

3 Guangdong Key Laboratory for Biomedical Measurements and Ultrasound Imaging, School of Biomedical Engineering, Health Science Center, Shenzhen University, Shenzhen, 518055, China

\section{*Correspondence to:}

Dr. Lin Guimiao,

School of Basic Medical Sciences, Shenzhen University Health Sciences Center, Shenzhen 518060, China

Phone: 86-755-86671903

Fax: 86-755-86671903

Email: gmlin@szu.edu.cn 


\begin{abstract}
:
Background: Indium phosphide (InP) quantum dots (QDs) have shown a broad application prospect in the fields of biophotonics and nanomedicine. However, the potential toxicity of InP QDs has not been systematically evaluated. In particular, the effects of different surface modifications on the biodistribution and toxicity of InP QDs is still unknown, which hinders their further developments. The present study aims to investigate the biodistribution and in vivo toxicity of three types of $\mathrm{InP} / \mathrm{ZnS}$ QDs with different surface modifications, hQDs (QDs-OH), aQDs (QDs-NH$)$, and cQDs (QDs$\mathrm{COOH}$ ) respectively. These $\mathrm{InP} / \mathrm{ZnS}$ QDs with $2.5 \mathrm{mg} / \mathrm{kg} \mathrm{BW}$ or $25 \mathrm{mg} / \mathrm{kg} \mathrm{BW}$ were intravenously injected into BALB/c mice. Biodistribution of three QDs was determined through cryosection fluorescence microscopy and ICP-MS analysis. The subsequent effects of InP/ZnS QDs on histopathology, hematology and blood biochemistry were evaluated at $1,3,7,14$ and 28 days post-injection.
\end{abstract}

Results: The results showed these types of InP/ZnS QDs QDs were rapidly distributed in the major organs of mice, mainly in the liver and spleen, and lasted for 28 days. No abnormal behavior, weight change or organ index were observed during the whole observation period, except that 2 mice died on Day 1 after $25 \mathrm{mg} / \mathrm{kg} \mathrm{BW}$ hQDs treatment. The results of H\&E staining showed that no obvious histopathological abnormalities were observed in the main organs (including heart, liver, spleen, lung, kidney, and brain ) of all mice injected with different surface functionalized QDs. Low concentration exposure of three QDs hardly caused obvious toxicity, while high concentration exposure of the three QDs could cause some changes in hematological parameters or biochemical parameters related to liver function or cardiac function. More attention need to be paid on cQDs as high dose exposure of cQDs induced death, acute inflammatory reaction and slight changes in liver function in mice.

Conclusions: Generally speaking, the surface modification and exposure dose can influence the biological behavior and in vivo toxicity of QDs. The surface chemistry should be fully considered in the design of InP-based QDs for their biomedical applications. 
Keywords: $\mathrm{InP} / \mathrm{ZnS}$ quantum dots, surface chemistry, in vivo, biodistribution, nanotoxicology 


\section{Background}

In the past few years, nanomaterials have attracted widespread interest owing to their unique magnetic, optical, thermal or conductive properties. Quantum dots (QDs), as a kind of interesting semiconductor nanomaterials, are composed of group II - IV or III - V elements with the diameter of 1-10 $\mathrm{nm}$. They are increasingly used in medical and pharmaceutical science due to their properties, such as broad absorption spectra, narrow tunable photoluminescence (PL) spectra, high quantum yield, light bleaching resistance, and large specific surface area $[1 ; 2]$. These advantages enable QDs to be used in the field of biological imaging, drug deliver and diagnostics after they are coupled with different biomolecules [3]. Despite the numerous benefits provided by QDs, people still have doubts about their potential harmful health effects, which are related to the heavy metals such as $\mathrm{Cd}, \mathrm{Pb}, \mathrm{As}$, and $\mathrm{Te}$ in these materials $[4 ; 5]$. Considering the inherent toxicity of Cd-based QDs, it is critical to find other kinds of safer QDs.

Among the Cd-free alternatives, indium phosphide (InP) QDs have shown great potential as a replacement for Cd-based QDs. Because InP QDs do not contain heavy metal elements, show stable quantum yield and size-tunable PL emission from visible to near infrared (NIR) range, InP QDs are beneficiary in biological applications $[6 ; 7]$. For example, Zhang et al carried out in vivo imaging of tumor bearing nude mice with silica medium composite probe encapsulated $\mathrm{InP} / \mathrm{ZnS}$ QDs, and found that the composite probe had excellent tumor targeting and fluorescence imaging capabilities [8]. More importantly, different from the ionic bond in CdSe QDs, the covalent bond in InP QDs is stronger and more robust, which make them less toxicity $[9 ; 10]$. Some studies have proved the biosafety of InP QDs. For instance,. Yaghini et al. found that InP QDs mainly accumulated in the liver and spleen of rats, with no abvious organ damage, histopathological lesions or serum biochemical changes when the rats were injected intravenously with InP QDs at the dose of $12.5 \mathrm{mg} / \mathrm{kg}$ body weight $(\mathrm{BW})$ or $50 \mathrm{mg} / \mathrm{kg} \mathrm{BW}$ [11]. Brunetti et al. found that the toxicity of $\mathrm{InP} / \mathrm{ZnS}$ QDs was much lower than that of $\mathrm{CdSe} / \mathrm{ZnS}$ QDs by comparing their toxicity in vitro and in vivo 
(animal model Drosophila), and they considered InP/ZnS QDs were safer alternatives to $\mathrm{CdSe} / \mathrm{ZnS}$ QDs [12]. However, some published studies have provided evidence of biological damage caused by InP QDs. For example, Yamazaki et al. investigated the survival of the Syrian golden hamster for two years, during which they were given 3 $\mathrm{mg} / \mathrm{kg}$ InP particles intratracheally twice a week for 8 weeks. Severe pulmonary inflammation and localized bronchioloalveolar cell proliferation was observed after the last administration [13]. Chen et al. reported InP/ZnS QDs could cause the deformation and death during the development of Chinese rare minnow embryos, although the effects were weaker than Cd-based QDs and CuInS/ZnS QDs [14].

As we all know, QDs are possibly exposed to organisms through many routes, including lung inhalation, ingestion, skin contact, and even intravenous injection during the process of production, application and wasting [15]. Then they may circulate, metabolize, excrete or accumulate in the body, and produce varing levels of biological toxic effects on organisms. The in vivo biological features of QDs mainly depend on the physicochemical characteristics such as elemental composition, particle size, surface charge, surface chemical properties $[16 ; 17 ; 18]$. Du et al. reported that PEG functionalization could reduce the accumulation of CdTe QDs in liver and kidney, and also decrease the oxidative stress variation [19]. Since the majority of QDs are composed of toxic substances, shells or macromolecules are often coated on the surface in order to reduce the biotoxicity [20;21]. In addition, researchers hope the QDs could be eliminated from the body over time, rather than being broken down or accumulated in organs for a long time. Thus, the in vivo distribution and toxicity of QDs with different physicochemical properties need to be deeply addressed before wide-scale biological application of QDs. However, there are limited reports on the toxicity of InP QDs, and most of them focus on toxicity studies using in vitro cell model or simple model organisms, which may hinder the biomedical application of InP QDs.

In the present study, in order to evaluate the in vivo distribution and biotoxicity of InP/ZnS QDs with different surface modifications, three kinds of commercially available InP/ZnS QDs, including hydroxylated QDs (hQDs), amino QDs (aQDs), and carboxylic QDs (cQDs) were injected intravenously into mice, respectively. Mice were 
sacrificed at 1, 3, 7, 14 and 28 days post-exposure. By comparing the behavior, weight, organ coefficient, hematological parameters, serum biochemical parameters, biodistribution and organ histopathology of the animal models in different groups, the effects of different surface functional groups on the toxicity of $\mathrm{InP} / \mathrm{ZnS}$ QDs were demonstrated. Our study may provide a better understanding of the effects of surface chemistry on QDs toxicity at the animal level and also facilitate the synthesis of safer QDs in biomedical applications.

\section{Results}

\section{Characterization of InP/ZnS QDs}

TEM was used to evaluate the shape, size and morphology of the three types of $\mathrm{InP} / \mathrm{ZnS}$ QDs with different surface functional groups (-OH, $-\mathrm{NH}_{2}$ and $\left.-\mathrm{COOH}\right)$. The representative images of hQDs, aQDs and cQDs were shown in Figure 1(A-C), respectively, suggesting that the three kinds of $\mathrm{InP} / \mathrm{ZnS}$ QDs were spherical or ellipsoidal, with uniform particle size of approximately 3-7 nm. The diameter of aQDs was slightly smaller than that of hQDs and cQDs. The hydrodynamic diameter and Zeta potential of these three water-soluble InP/ZnS QDs were investigated by zeta potential and particle size analyzer. The results were shown in Figure 1(D-F) and Table 1. The hydrodynamic diameter of hQDs, aQDs and cQDs was $(51.01 \pm 1.29) \mathrm{nm},(50.43 \pm 2.03)$ $\mathrm{nm}$, and $(47.36 \pm 1.85) \mathrm{nm}$, respectively. The Zeta potential of the three water-soluble $\mathrm{InP} / \mathrm{ZnS}$ QDs was $-(21.03 \pm 0.61) \mathrm{mV},-(21.63 \pm 1.07) \mathrm{mV}$, and $-(22.67 \pm 0.15) \mathrm{mV}$. Optical properties of QDs were also evaluated and the results were shown in Figure 1(G-I). Evaluation of the absorbance spectra indicated the first exciton peak of the three $\mathrm{InP} / \mathrm{ZnS}$ QDs was around at $350 \mathrm{~nm}$. From the results of fluorescence spectra, the emission spectra of the three water-soluble QDs were narrow and symmetrical, and the emission peak was about at $(625 \pm 10) \mathrm{nm}$ with the excitation at $380 \mathrm{~nm}$. 
Table 1. Particle size, hydrodynamic diameter and Zeta potential of these QDs

\begin{tabular}{cccc}
\hline QDs & Particle size $(\mathrm{nm})$ & Hydrodynamic diameter $(\mathrm{nm})$ & Zeta potential $(\mathrm{mV})$ \\
\hline hQDs & $5.94 \pm 1.08$ & $51.01 \pm 1.29$ & $-(21.03 \pm 0.61)$ \\
aQDs & $3.39 \pm 0.80$ & $50.43 \pm 2.03$ & $-(21.63 \pm 1.07)$ \\
cQDs & $5.12 \pm 0.74$ & $47.36 \pm 1.85$ & $-(22.67 \pm 0.15)$ \\
\hline
\end{tabular}

A
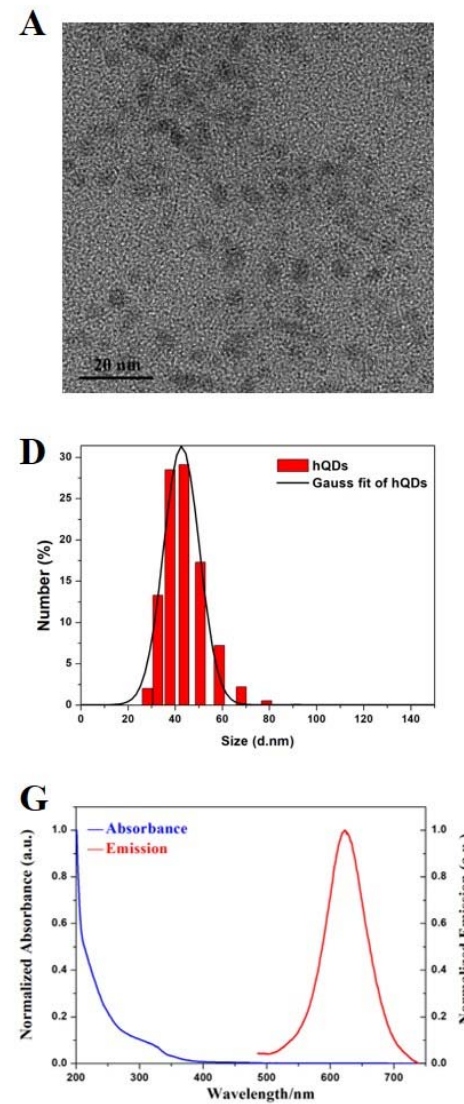

B
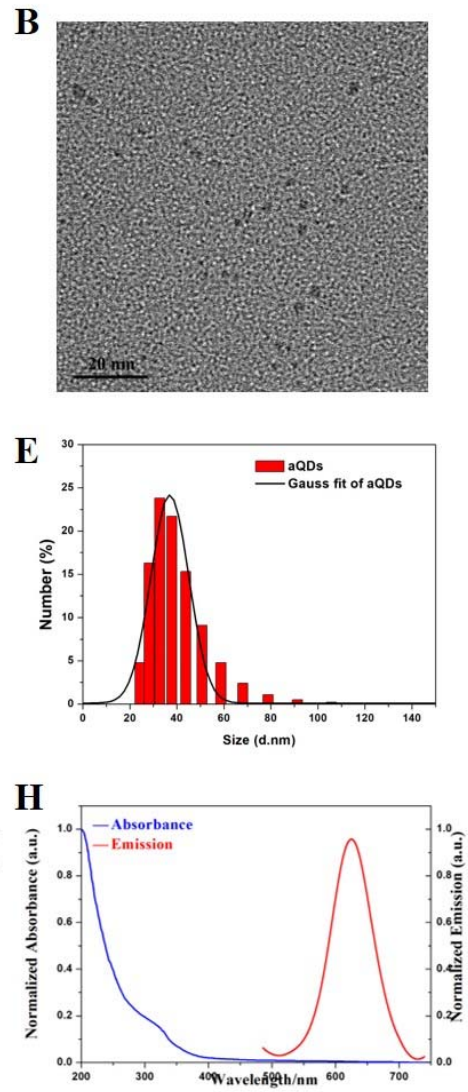

C
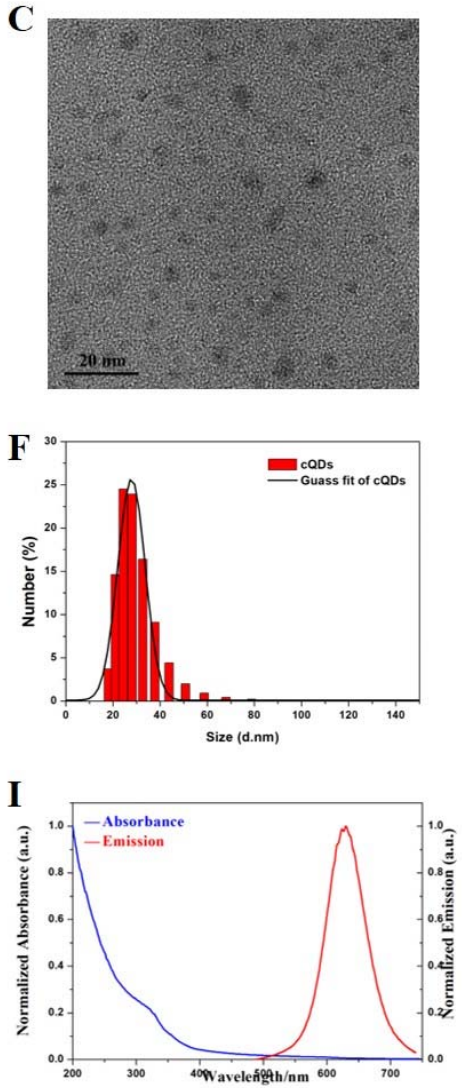

Figure 1. Characterization of InP/ZnS QDs with different surface modifications. (A-C) TEM image of hQDs, aQDs, and cQDs, respectively. (D-F) DLS of hQDs, aQDs and cQDs dispersed in deionized water. (G-I) Normalized absorption spectra and normalized PL spectra of hQDs, aQDs and cQDs, respectively

\section{Body weight and organ weight/BW coefficients}

The behavior, mental status, food intake, urine and feces of mice were observed daily after intravenous injection of QDs. Two mice from cQDs-treated group died on 
the first day after treatment. Besides, no unusual changes were observed in food intake, fur, behavior and mental status after exposure of QDs. The body weight of mice was continuously recorded for 28 days and the data was shown in Figure 2A. The body weight of QDs-exposed groups and the control group showed comparable increasing trends through the study. Main organs of mice were removed and weighted carefully when the mice were sacrificed at various post-injection times. Organ index of the main organs was calculated as organ weight $(\mathrm{mg}) / \mathrm{BW}(\mathrm{g})$. The organ index of heart, liver, spleen, lung, kidney and brain of mice on Day 28 were shown in Figure 2B, there was no significant statistical difference in all organ index between QDs treated groups and control group. The above results suggested that these InP/ZnS QDs did not interfere with the growth of mice.
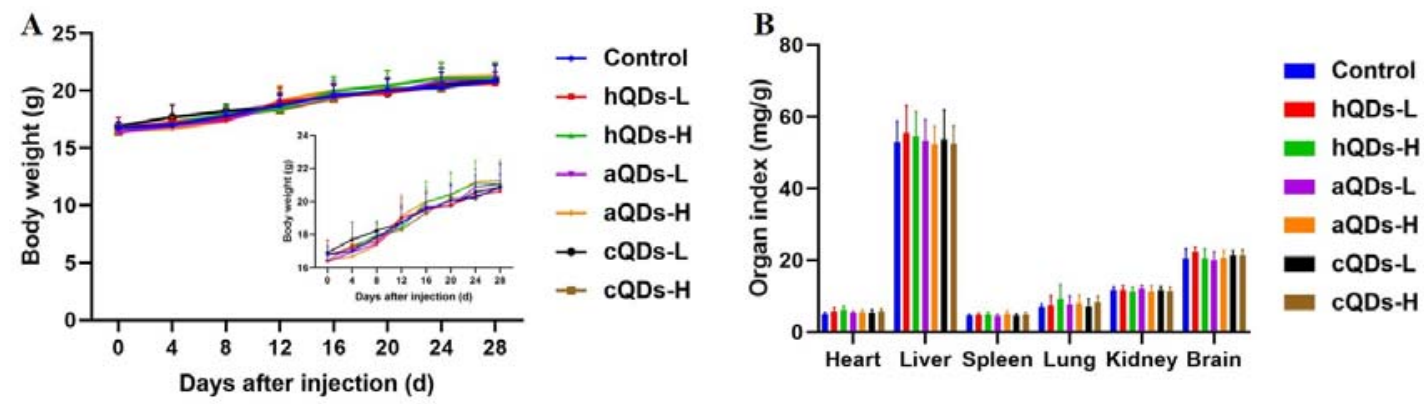

Figure 2. Body weight and organ index in mice. (A) The body weight curve of mice continuously monitored for 28 days (Inset showed the magnified state of the body weight curve). (B) The main organ index of mice on Day 28 after administration.

\section{In vivo distribution of QDs}

After QDs enter the body through intravenous injection, they will be transported to various organs along with the blood stream. The distribution of QDs in main organs was observed by cryosection fluorescence microscopy. Fluorescence images of different tissues from mice treated with $25 \mathrm{mg} / \mathrm{kg} \mathrm{BW}$ hQDs, aQDs and hQDs at indicated time points were shown in Figure (3-5), respectively. Strong fluorescence of QDs could be observed in almost all tissue sections. Especially in the liver and spleen, QDs exhibited a bright and uniform punctate pattern. The PL intensity appeared to be 
weakening over time although the fluorescence signal still maintained in livers, spleens and kidneys. It should be noted that no fluorescence was observed in the tissue sections of the control group (data not shown). The results suggested that the three kinds of QDs could accumulate in the major organs of mice and keep their unique fluorescence characteristics at least 28 days. Though very little fluorescence was observed in brain tissue sections after mice were exposed to QDs, it indicated that all three QDs could pass through the blood-brain barrier and distribute in the brain.

In order to further quantitate the accumulation of three kinds of QDs in liver, kidney and spleen, the In element concentration in tissues at different sampling times was measured by ICP-MS. The results were shown in Figure 6. The three QDs were mainly distributed in liver and spleen, and this result was consistent with that of cryosection fluorescence microscopy. The concentration of In in kidney was about one tenth of that in liver and spleen. The accumulation of three QDs in the liver reached the peak on 3 days post-injection (hQDs: $7.95 \mu \mathrm{g} / \mathrm{g}$ In, aQDs: $9.06 \mu \mathrm{g} / \mathrm{g}$ In, and cQDs: 6.92 $\mu \mathrm{g} / \mathrm{g}$ In). The distribution of aQDs and cQDs in spleen also reached the peak on Day 3 after administration, but In concentration of hQDs peaked in spleen at 7 days postinjection. After that, the In concentration in liver, spleen and kidney decreased gradually. However, In could be still detected in liver, spleen and kidney tissues from mice treated with high-dose QDs on Day 28. Since In was not detected in all tissues of the control group, it could be considered that all detected In element in liver, spleen and kidney came from the residues of QDs in vivo. The above results showed that the three QDs (hQDs, aQDs, and cQDs) were mainly distributed in the liver and spleen when they were injected intravenously. Although QDs may be removed or broken down in vivo, this process will take quite a long time. 


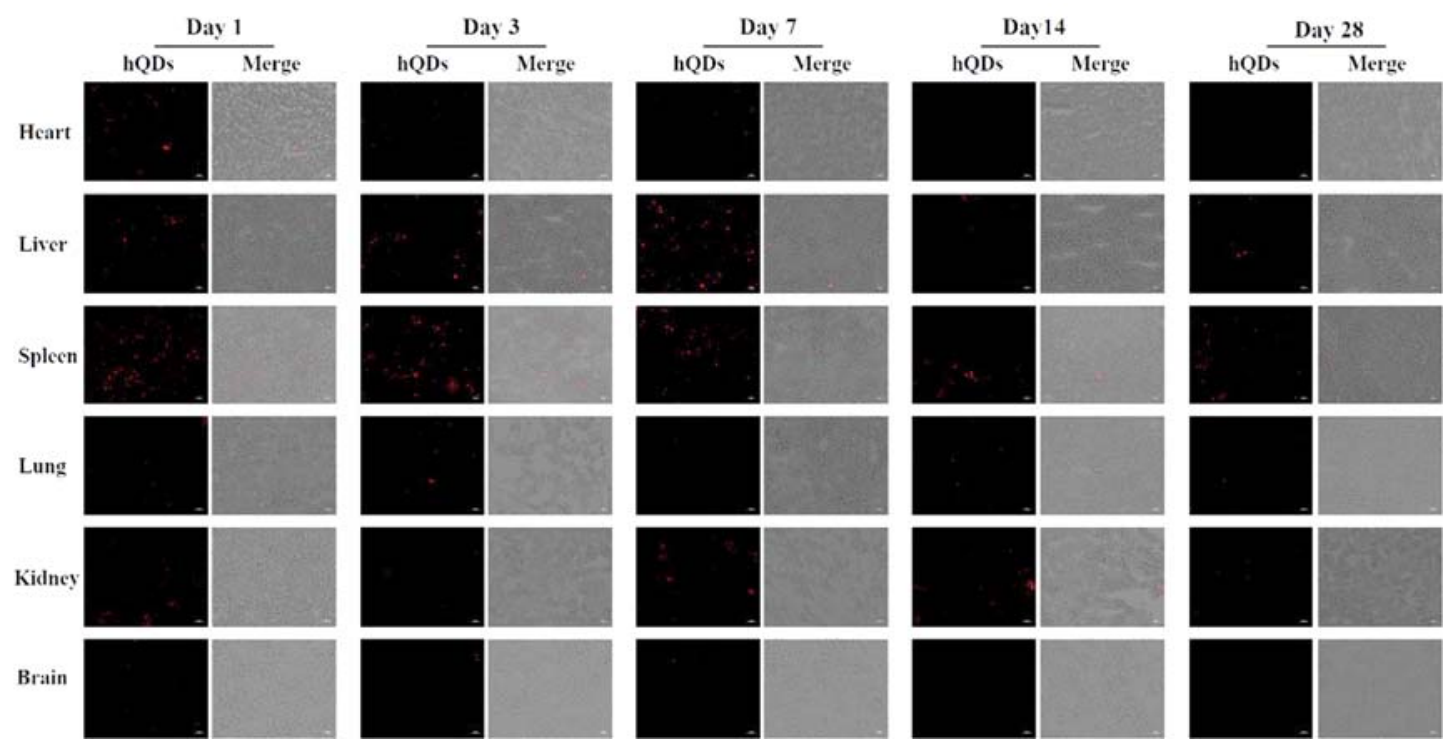

Figure 3. Fluorescence images of main organ tissues of mice on Day1, Day 3, Day

7, Day 14 and Day 28 after treated with $25 \mathrm{mg} / \mathrm{kg}$ BW hQDs (scale bar: $50 \mu \mathrm{m}$ ).

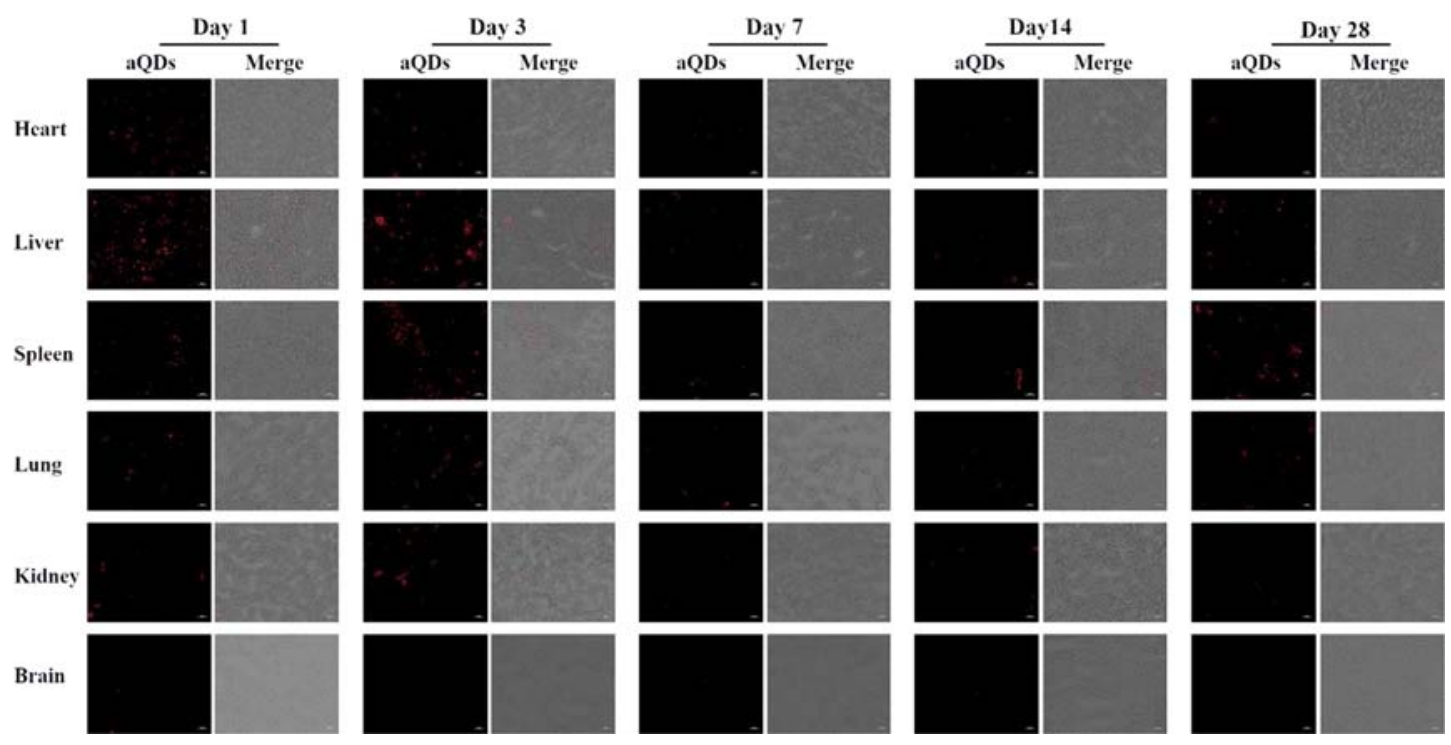

Figure 4. Fluorescence images of main organ tissues of mice on Day1, Day 3, Day

7, Day 14 and Day 28 after treated with $25 \mathrm{mg} / \mathrm{kg} \mathrm{BW}$ aQDs (scale bar: $50 \mu \mathrm{m}$ ). 

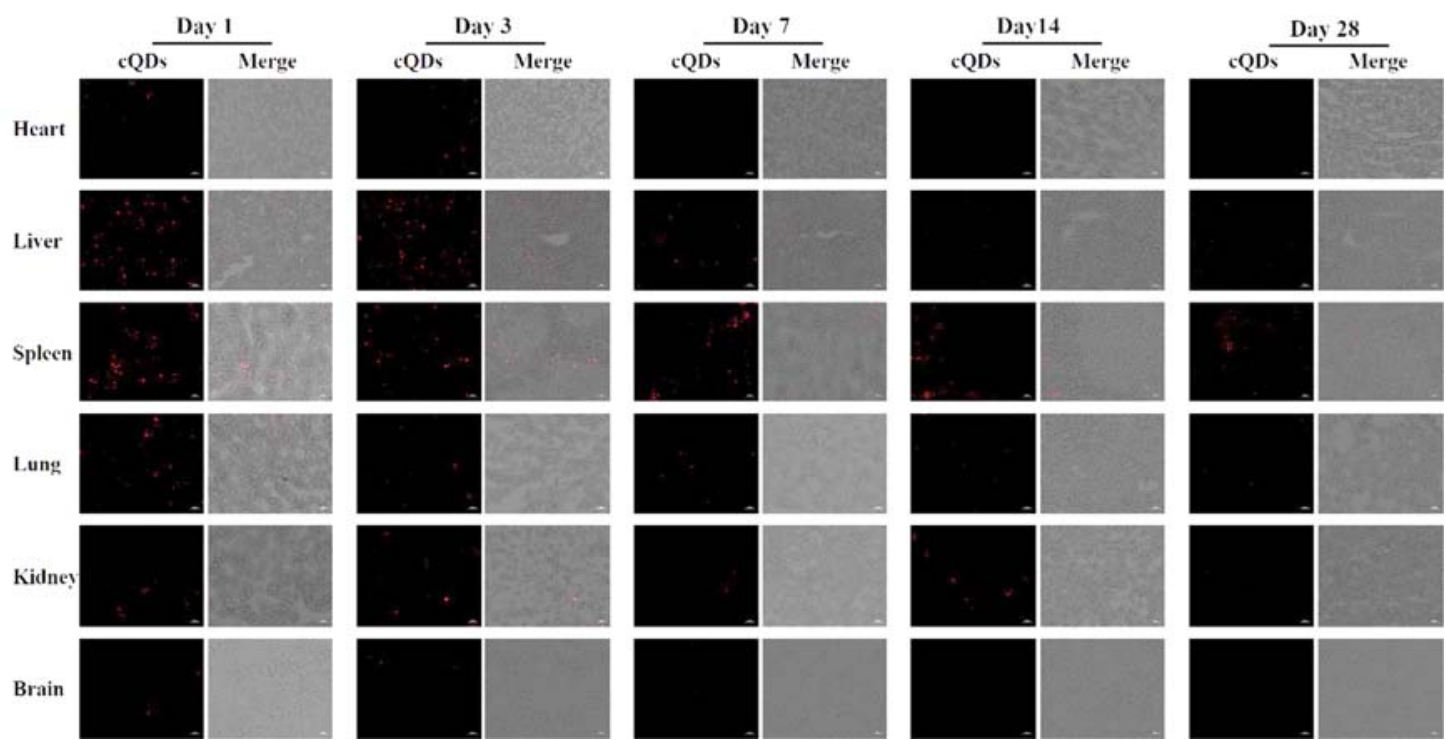

Figure 5. Fluorescence images of main organ tissues of mice on Day1, Day 3, Day

7, Day 14 and Day 28 after treated with $25 \mathrm{mg} / \mathrm{kg}$ BW cQDs (scale bar: $50 \mu \mathrm{m}$ ).
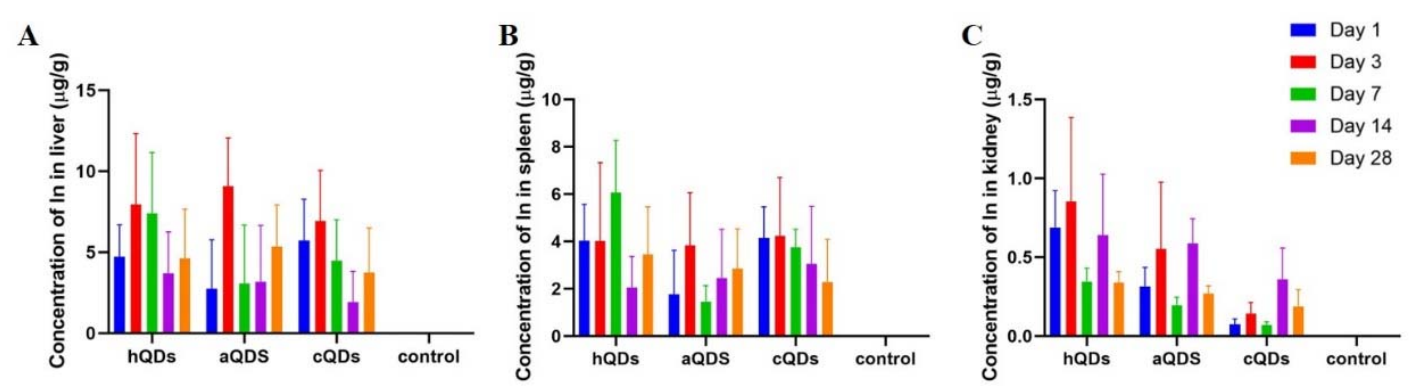

Figure 6. The In element concentration in organs over a period of 28 days in mice following administration of three $\mathrm{InP} / \mathrm{ZnS}$ QDs at $25 \mathrm{mg} / \mathrm{kg}$ BW. (A) Liver tissues. (B) Spleen tissues. (C) Kidney tissues.

\section{Histopathological detection results}

Histological assessment was performed to evaluate the tissue damage and inflammation caused by QDs exposure. Main organs of all mice were sliced into $5 \mu \mathrm{m}$ sections and stained with $H \& E$. The representative histological results were shown in Figure 7. After mice were intravenously injected different surface functionalized QDs at the dose of $25 \mathrm{mg} / \mathrm{kg}$ BW or $2.5 \mathrm{mg} / \mathrm{kg} \mathrm{BW}$, no sign of inflammatory response or pathological changes were observed in heart, liver, spleen, lung, kidney, and brain of all mice at different sampling times when compared with the control group. Therefore, 
although all the three kinds of QDs could remain in the organs of mice, especially in liver and spleen for a long time, no obvious histopathological abnormalities were observed, which suggested $\mathrm{InP} / \mathrm{ZnS}$ QDs with different surface functional groups (-OH, $-\mathrm{NH}_{2}$, and $\left.-\mathrm{COOH}\right)$ caused low toxicity to these organs.

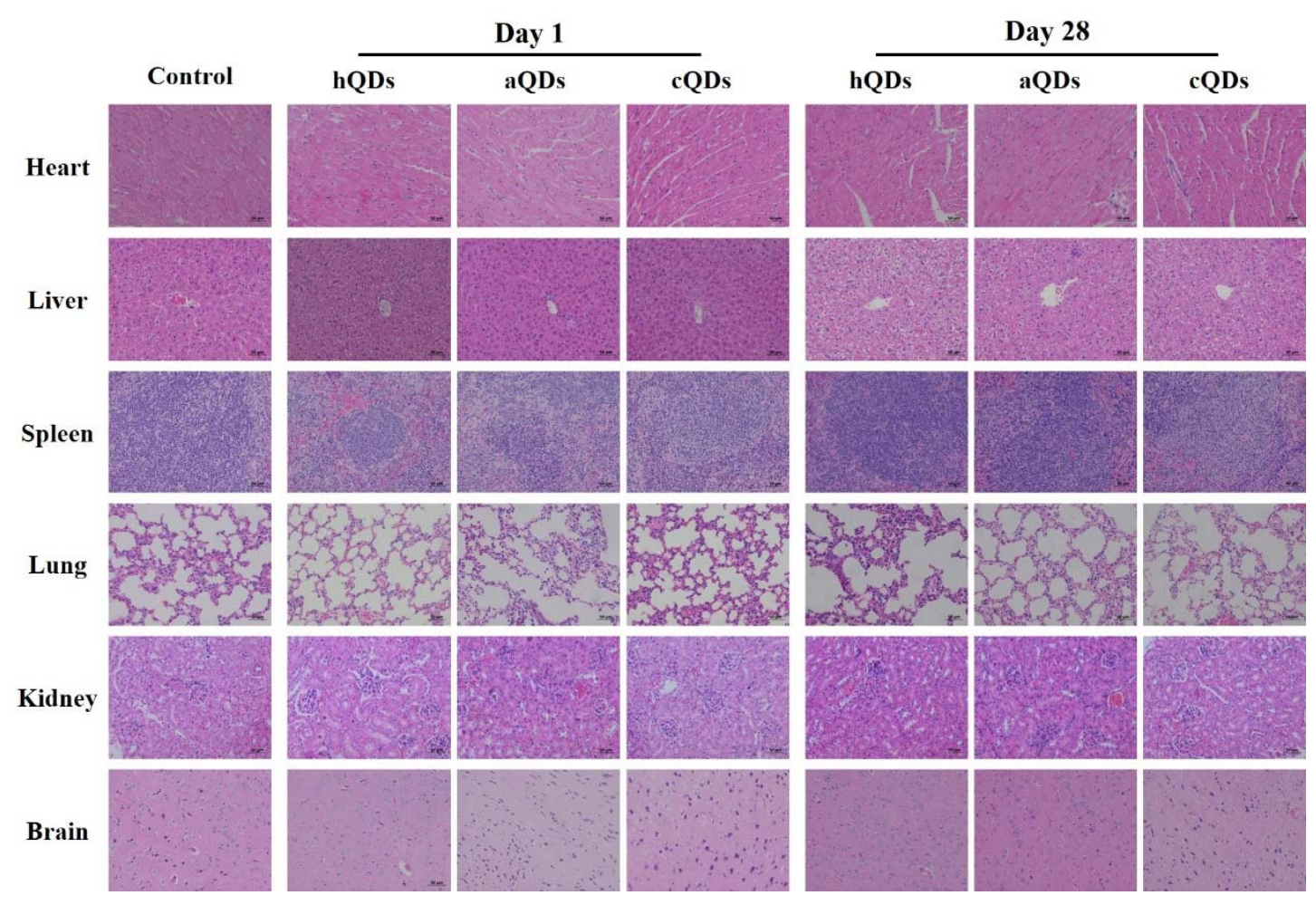

Figure 7. Representative histological images of major organs including heart, liver, spleen, lung, kidney and brain collected from the control group mice and different surface functionalized QDs treated mice following intravenous injection at dose of 25 $\mathrm{mg} / \mathrm{kg}$ at 1 day and 28 days post-injection (scale bar: $50 \mu \mathrm{m}$ ).

\section{Hematology analysis}

Since the three QDs will remain in vivo for a long time, the changes of blood cells can reflect some pathological reactions induced by QDs. Routine Blood analysis was performed and the results were shown in Figure 8. Most of hematological parameters obtained from QDs-treated mice were comparable to those in control group. On the first day after treatment, WBC counts in aQDs and cQDs group were obviously higher than that in control group $(P<0.05)$, which indicated that these two QDs may cause an acute 
inflammatory response after entering the body. The percent of Nue and Lym on Day 3 and Day 28 in aQDs groups was significantly higher than that in control group $(P<$ 0.05). The levels of RDW-CV on Day 3, PCT and MPV on Day 7 in hQDs group were remarkabley higher than those in control group $(P<0.05)$. The levels of PLT, PCT and MPV in cQDs groups were obviously changed compared to those of the control group $(P<0.05)$. No significant differences were found in the other parameters. The above results showed that a high dose of the three QDs caused changes in hematological indexes. In particular, aQDs and cQDs could cause acute inflammation in the body, hQDs mainly had adverse effects on red blood cells and platelets.
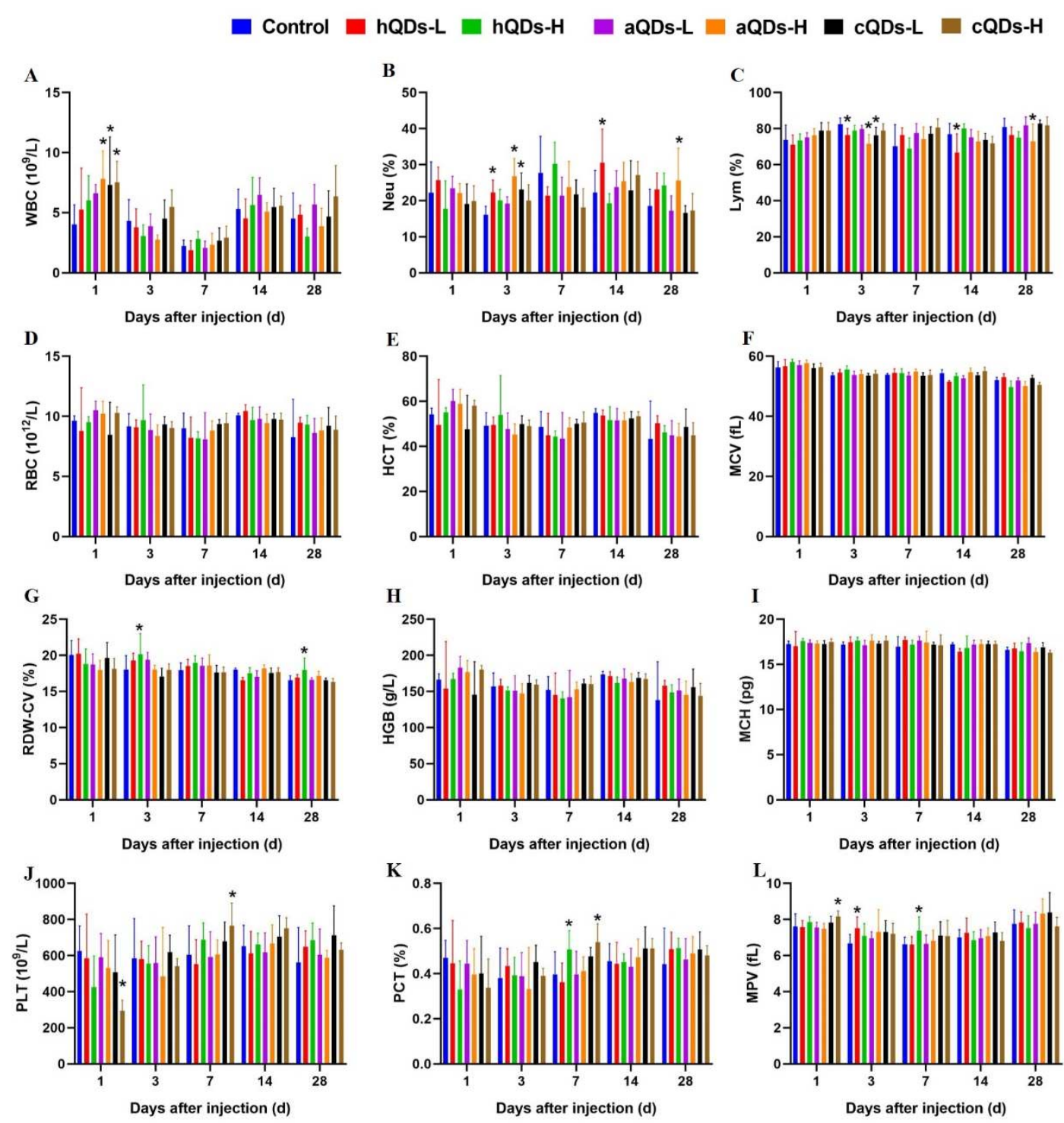

Figure 8. Hematology results of the control group mice and different surface functionalized QDs treated mice following intravenous injection at 1, 3, 7, 14 and 28 
days post-injection. (A) White blood cell (WBC). (B) Neutrophils (Neu) percentage. (C) Lymphocyte (Lym) percentage. (D) Red blood cell (RBC) count. (E) Hematocrit (HCT) levels. (F) Mean corpuscular volume (MCV) levels. (G) Coefficient of variation of $\mathrm{RBC}$ volume distributing width (RDW-CV) levels. (H) Hemoglobin concentration (HGB) levels. (I) Mean corpuscular hemoglobin (MCH) levels. (J) Platelet (PLT) count. (K) Thromboplastin (PCT) levels. (L) Mean platelet volume (MPV) levels. (*Significantly different compared to control group at the same sampling time, $P<$ 0.05.)

\section{Serum biochemical analysis}

Serum biochemistry tests were performed to observe the effects of InP/ZnS QDs on biological functions of major organs. Liver function indexes including ALT, AST, ALP, TP, ALB, GLB, A/G, T-Bil, D-Bil, TG, TC, HDL-C, LDL-C, GLU were measured and the results were presented in Figure 9(A-N). In high dose hQDs treated groups, levels of TG and TC at 1, 3, 14 and 28 days post-injection were significantly higher than that in control group $(P<0.05)$. Levels of T-Bil, D-Bil and LDL-C on Day 1, TP and HDL-C on Day 3, ALT and T-Bil on Day 14 were also obviously higher than that in control group $(P<0.05)$. In high dose aQDs treated groups, levels of ALP, A/G, DBil and LDL-C on Day1, ALP, ALB, TC on Day 3, TC on Day 28 were remarkably higher than that in control group $(P<0.05)$. In high dose cQDs treated groups, LDL-C on Day 1, A/G on Day 3 and GLU on Day 28 were obviously higher than that in control group $(P<0.05)$. Almost all the low dose QD exposed groups showed no significant changes in the above biochemical indexes. The results showed that the three surface functionalized InP/ZnS QDs could affect the liver function of mice when exposed at high concentration, the effect of hQDs was the most serious, and the effect of aQDs and cQDs was relatively mild.

Heart function indexes including LHD, CK, CK-MB and $\alpha-\mathrm{HBDH}$ of all mice were measured and the results were shown in Figure 9(O-R). Levels of LDH on Day 3 and Day 28, $\alpha-\mathrm{HBDH}$ on Day 3 in high dose hQDs treated group were significantly higher than that in control group $(P<0.05)$. Levels of $\alpha-\mathrm{HBDH}$ on Day 7 and LDH on 
Day 14 in high dose aQDs treated group were obviously higher than that in control group $(P<0.05)$. There were no significant differences in $\mathrm{CK}$ and $\mathrm{CK}$ MB levels between QDs treated groups and control group. The above results showed that the hQDs and aQDs could cause slight effects on cardiac function, while cQDs had almost no effect on cardiac function. Furthermore, renal function indexes including UA, Urea and CREA of all mice were also detected, and the results were shown in Figure9 (S-U). There were no significant changes in UA, Urea and CREA levels of the three QDs treated mice when compared with the control groups at the same sampling time. It illustrated that the three QDs with different surface functional groups had no toxicity to the kidney. 
Control $\square$ hQDs-L hQDs-H a aQDs-L aQDs-H a cQDs-L $\square$ cQDs-H
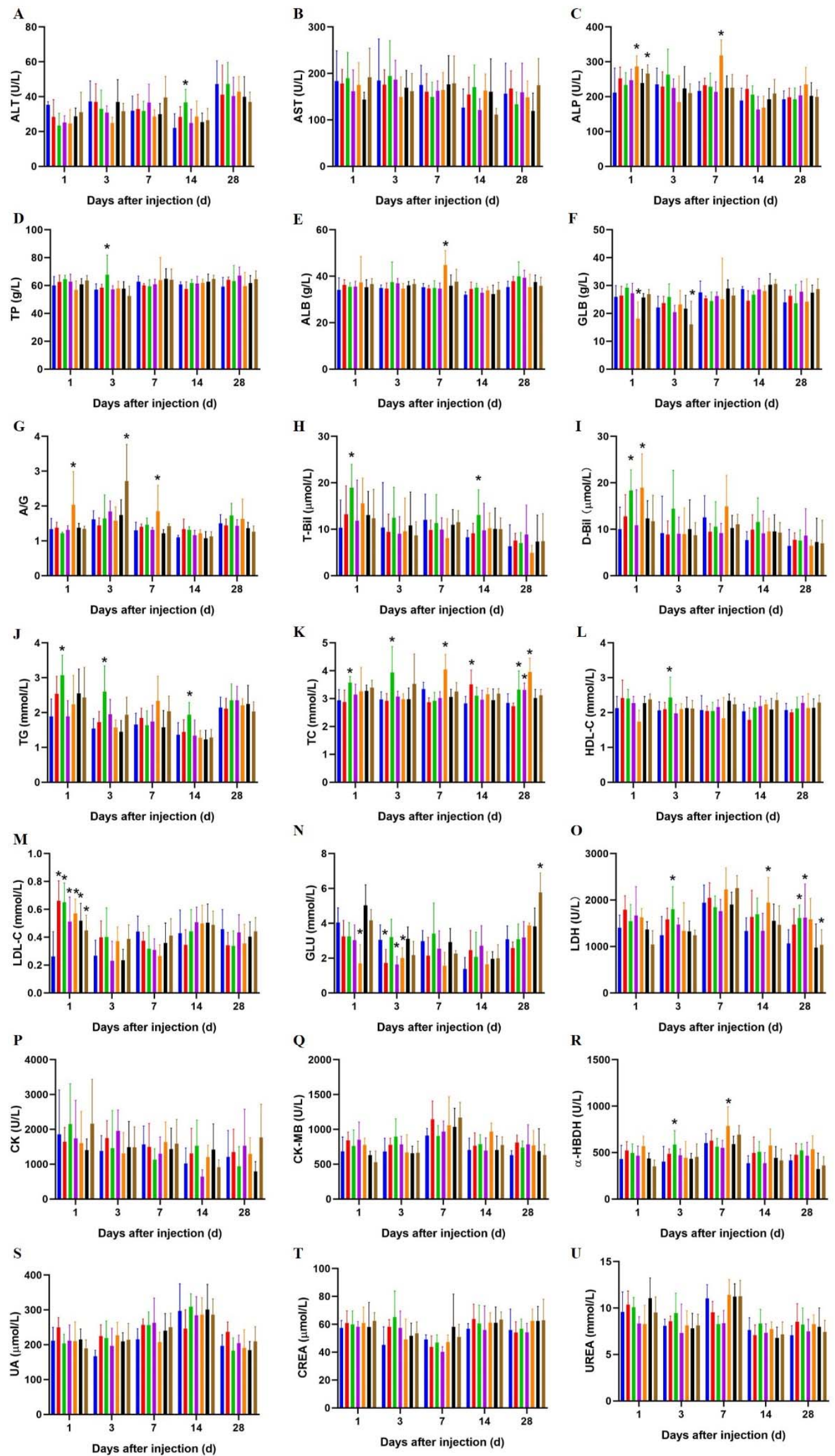
Figure 9. Serum biochemical results of the control group mice and different surface functionalized QDs treated mice following intravenous injection at 1, 3, 7, 14 and 28 days post-injection. (A) Alanine aminotransferase (ALT) levels. (B) Aspartate transaminase (AST) levels. (C) Alkaline phosphatase (ALP) levels. (D) Total protein (TP) levels. (E) Albumin (ALB) levels. (F) Globulin (GLB) levels. (G) Albumin and globulin ratio (A/G). (H) Total bilirubin (T-Bil) levels. (I) Direct bilirubin (D-Bil) levels. (J) Triglyceride (TG) levels. (K) Total cholesterol (TC) levels. (L) High density lipoprotein (HDL-C) levels. (M) Low-density lipoprotein (LDL-C) levels. (N) Glucose (GLU) levels. (O) Lactate dehydrogenase (LDH) levels. (P) Creatine kinase (CK) levels. (Q) Creatine kinase isoenzyme (CK-MB) levels. (R) $\alpha$-Hydroxybutyrate dehydrogenase ( $\alpha$-HBDH) levels. (S) Uric acid (UA) levels. (T) Urea levels. (U) Creatinine (CREA) levels. (*Significantly different compared to control group at the same sampling time, $P<0.05$.)

\section{Discussion}

QDs are of great interest due to their unique optical and electronic properties, and have shown great potential applications in fluorescent probes, bioimaging, gene delivery and biosensors. However, the inherent toxicity of restricted heavy metals such as $\mathrm{Cd}, \mathrm{Pb}$, et al. severely hinders the clinical translation of QDs. Cd-free QDs alternatives are being widely investigated and InP QDs have shown a great potential as a replacement for CdSe QDs in applications. Therefore, it is vital to understand the biological fate and toxicity of InP QDs for its successful application in biological science. The toxicity of nanomaterials can be evaluated by both in vitro model and in vivo system. Up to now, there are very few studies on the toxicity of $\mathrm{InP} / \mathrm{ZnS}$ QDs, which are carried out by in vitro cell lines [22] or simple model organisms (Hydra vulgaris [23], rare minnow embryos [24]). As the biological aspects of animal models are very similar to human biology, it is an ideal model to systematically evaluate the toxicology of nanomaterials. As we all know, the in vivo toxicity of QDs is related to many factors, such as dosage, route of administration, exposure time, immune clearance ability of the body, et al.. In terms of QDs themselves, the chemical physical properties 
(such as chemical composition, size, shape, surface charge, surface modification, outer inorganic and organic shells etc.) determine their efficacy and toxicity to a large extent. It is very important to comprehensively evaluate the disposition of nanoparticles in vivo, so as to understand and predict their effectiveness and side effects [25]. In this study, we systematically studied the tissue biodistribution and in vivo toxicity of InP/ZnS QDs with different surface modifications $\left(-\mathrm{OH},-\mathrm{NH}_{2},-\mathrm{COOH}\right)$ and different doses $(2.5$ $\mathrm{mg} / \mathrm{kg} \mathrm{BW}, 25 \mathrm{mg} / \mathrm{kg} \mathrm{BW}$ ) in BALB/c mice after a single intravenous injection, and we hope to fill in the gap of $\mathrm{InP} / \mathrm{ZnS}$ QDs toxicity in vivo.

The biodistribution of QDs and the concentration of heavy metals released by QDs are the key parameters to evaluate their toxicity in vivo. Several studies have reported the biodistribution of different types of Cd-based QDs in mice or rat models. Haque et al reported that the fluorescence of CdSe/CdS-MPA QDs was able to be observed in the liver, spleen, lung and kidneys after repeated intraperitoneal injection into BALB/c mice. No histopathological changes were observed, but the level of IL-6 increased in plasma, liver and spleen [26]. In this study, cryosection fluorescence microscopy and ICP-MS was utilized to detect the distribution of three surface functionalized $\mathrm{InP} / \mathrm{ZnS}$ QDs in mice after a single intravenous injection at $25 \mathrm{mg} / \mathrm{kg} \mathrm{BW}$. Strong fluorescence of the three QDs could be observed in almost all tissue sections of main organs at 1 day post-injection. It suggested that all the three QDs could distribute in heart, liver, spleen, kidney, lung and brain along with blood circulation and kept their unique fluorescence characteristics. QDs were excreted or broken down over time, but their fluorescence could still be observed in the liver and spleen on Day 28. The results of ICP-MS were consistent with those of fluorescence imaging. The three QDs were mainly distributed in liver and spleen. The concentration of In in kidney was about one tenth of that in liver and spleen. When nanoparticles entered the physiological environment, their physical and chemical properties or integrity may change significantly following protein binding, biomolecular corona formation or cell internalization [27; 28]. Liu N et al reported that $\mathrm{CdTe} / \mathrm{ZnS}$ QDs were chemically stabilized and could not elicit biological responses in vitro, but they would undergo degradation in vivo, since the $\mathrm{Cd}$ :Te ratio gradually decreased with time compared with the intact CdTe/ZnS QDs 
from 6 hours after injection [29]. Some published research have reported Cd-based QDs can lead to reduced cell survival, production of reactive oxygen species (ROS), DNA damage, mitochondrial damage or cell death in multiple cell lines [30;31], and can induce liver morphological and functional impairments, lung inflammation and injury in vivo experiments $[32 ; 33]$. As $\mathrm{Cd}^{2+}$ can be released via the oxidation of QDs, and then bound to sulfhydryl groups on proteins in cells, which leads to the functional degradation of various subcellular organelles, the release of $\mathrm{Cd}^{2+}$ is recognized as one of the main reasons for the toxicity of Cd-based QDs [34]. Compared with II-VI semiconductors, III-V semiconductors nanocrystals (particularly InP) have better structural robustness and stability which are attributed to the presence of covalent bonds in their matrix. Since the covalent bonding degree in InP nanocrystals is greater than that in II-VI semiconductors such as CdSe and CdTe, InP QDs are less likely to be degraded and release heavy metals in biological systems $[9 ; 10]$.

In this study, the fluorescence of QDs appeared to be weakening over time, but it could still be observed on 28 days post-injection, indicating that there were still intact QDs in tissues. There are two possible reasons for the weakening of fluorescence. Firstly, the integrity of QDs was broken after they entered the body because of the corrosive internal environment [35]. Once the QDs were destroyed, the surface trap states produced on the particles and affected the electron hole recombination process, and eventually lead to the decrease or disappearance of fluorescence intensity. Although the structural stability of InP QDs is better than Cd-based QDs, a recent study by Veronesi has reported that InP core was rapidly degraded in Hydra tissues without evident toxicity [36]. Another reason is that QDs were excreted through kidneys, bile ducts, lungs, secretory glands or other organs. It is generally believed that nanoparticles with a diameter less than $3 \mathrm{~nm}$ can extravasate into tissues nonspecifically. Nanoparticles with a diameter less than $5.5 \mathrm{~nm}$ can be excreted via renal clearance rapidly and efficiently, while the nanoparticles with a diameter more than $15 \mathrm{~nm}$ are hardly cleared via the urinary excretion $[37 ; 38]$. As the fluorescence signal of cryosection fluorescence microscopy is easy to be interfered by environmental factors and tissue auto fluorescence, In concentration of the three organs (liver, spleen and 
kidney) with the larger amount of QDs distribution was detected by ICP-MS on the basis of fluorescence imaging to reflect the existence of QDs in tissues. The In concentration of the three QDs reached the peak in liver and spleen 3-7 days after entering the body, but there were two peaks in the kidney, Day 3 and Day 14. It showed that a portion of QDs was excreted through renal clearance in the first three days after injected into body. Some portion of QDs were driven to kidney along with body fluids circulation and then excreted by the kidney. The three QDs still remained in the tissues on Day 28 from the results of ICM-MS and fluorescence imaging, which revealed both the excretion and degrading of QDs need quite a long time. Fitzpatrick et al. reported the fluorescence of CdSe/ZnS QDs could be retained and existed in nude mice for up to two years using both whole-body and microscopic fluorescence techniques [39]. Yaghin et al. reported the carboxylated In-based QDs could remain in liver and spleen tissues of rat for up to 90 days after intravenous injection at a dose of $12.5 \mathrm{mg} / \mathrm{kg} \mathrm{BW}$ [11].

Surface functionalization of nanoparticles is one of the critical steps to improve their physical and chemical properties for better applications. The change itself may bring potential risks and cause harm to the biological system [40; 41]. InP QDs are recognized as the low-toxic or non-toxic substitutes for Cd-based QDs. It is necessary to evaluate the safety of InP QDs with different surface modifications before practical applications. It is worth noting that $\mathrm{InP} / \mathrm{ZnS}$ QDs with different surface functional groups $\left(-\mathrm{OH},-\mathrm{NH}_{2}\right.$, and $\left.-\mathrm{COOH}\right)$ could exist in vivo for quite a long time from both the results of ICP-MS and fluorescence imaging, more attention should be paid to the toxic reaction caused by their distribution and accumulation. In this study, the toxicity of three InP/ZnS QDs with different surface functional groups were systematically investigated in mice following intravenous injection at either $2.5 \mathrm{mg} / \mathrm{kg}$ or $25 \mathrm{mg} / \mathrm{kg}$ $(n=7)$. Histopathology of main organs, the hematological indexes and biochemical indexes of all mice were performed at different predetermined time (at 1, 3, 7, 14, and 28 days post-injection). Throughout the whole study, besides two mice died on Day 1 after treated with $25 \mathrm{mg} / \mathrm{kg}$ cQDs, no unusual behavior or responses were observed in QDs treated mice compared to control mice. The results of histopathological 
examination showed that no obvious histopathological abnormalities were observed in the main organs (including heart, liver, spleen, lung, kidney, and brain ) of all mice at different sampling time after intravenously injected different surface functionalized QDs. From the results of hematology analysis, high dose of aQDs and cQDs could cause acute inflammation in the body, while hQDs mainly had adverse effects on red blood cells and platelets. From the results of biochemical analysis, the three QDs could affect the liver function of mice, the effect of hQDs was the most serious, and the effect of aQDs and cQDs was relatively mild. High dose of hQDs and aQDs could cause slight effects on cardiac function, while cQDs had almost no effect on cardiac function. No renal function was changed in all mice after exposed the three QDs. Moreover, the three $\mathrm{InP} / \mathrm{ZnS}$ QDs with exposure concentration of $2.5 \mathrm{mg} / \mathrm{kg} \mathrm{BW}$ showed no obvious toxicity on liver function, renal function and cardiac function in mice at different sampling times. Although the long-term accumulation of the three QDs with high exposure dose in vivo caused the changes of biochemical indexes of liver function and cardiac function, it did not cause histopathological abnormalities to the main organs, nor significantly change the growth and living habits of mice.

\section{Conclusions}

InP/ZnS QDs are considered as safer alternatives to Cd-based QDs. However, it is still lack of systematic and comprehensive observation and studies about biological distribution and in vivo toxicity of $\mathrm{InP} / \mathrm{ZnS}$ QDs. This work presents a detailed study of the toxicology and biodistribution of three $\mathrm{InP} / \mathrm{ZnS}$ QDs modified with -OH, $-\mathrm{NH}_{2}$ or $-\mathrm{COOH}$ groups respectively in mice following intravenous tail vein injection. Following administration, the three kinds of $\mathrm{InP} / \mathrm{ZnS}$ QDs could be rapidly distributed into the main organs of mice, especiallyin the liver and spleen. The three QDs were excreted from the body gradually, but the In element from QDs still could be detected in liver, spleen and kidney over 28 days period. Low concentration exposure of three QDs hardly caused obvious toxicity, while high concentration exposure of the three QDs could cause some changes in hematological parameters or biochemical parameters, and had no effect on histopathological changes. It should be noted that high dose 
exposure of cQDs could lead to death, acute inflammatory reaction and slight changes in liver function in mice, which was considered to be more toxic compared with the other two QDs. In conclusion, InP/ZnS QDs could be distributed to the main organs in vivo for a long time. Different surface modifications are crucial to the in vivo toxicity of QDs, which need to be taken into consideration in the synthesis and application of QDs in the future. Even though it will take quite a long time to realize the clinical transformation of QDs, especially in human body, we still hope that QDs can give full play to their excellent optical properties and drug delivery capacity in biological application with the rapid development of chemical synthesis technology, nano application and toxicology technology.

\section{Methods}

\section{Characterization of InP/ZnS QDs}

InP/ZnS QDs used in this study were prepared by Najing tech Company, China. Prior to be used in our experiments, the surface of QDs was modified with hydroxyl, amino and carboxyl groups, respectively. Finally, three water-soluble InP/ZnS QDs, including hydroxylated QDs (hQDs), amino QDs (aQDs), and carboxylic QDs (cQDs) were obtained. The size and morphology of the three QDs were characterized by transmission electron microscopy (TEM) (HT7700, HITACHI, Japan). Zeta potential and hydrodynamic size distribution of the three QDs was characterized by zeta potential and particle size analyzer (Brookhaven Instruments Inc., USA). Fluorescence spectra and absorption spectra were measured by fluorescence spectrophotometer (F-4600, HITACHI, Japan) and UV/Vis spectrophotometer (DU720, Beckman Coulter Inc., USA), separately. Moreover, the concentration of In in the three InP/ZnS QDs solution was quantitatively measured by inductively coupled plasma mass spectrometry (ICPMS, PerkinElmer, USA) and the exposure dose of QDs were calculated based on the mass concentration of the In.

\section{Animals}

Healthy female BALB/c mice (six weeks old) were purchased from Medical Laboratory Animal Center of Guangdong Province. The mice were raised in a ventilated, 
temperature-controlled and standardized sterile animal room with a 12-hour day/light circle at Shenzhen University. The mice were allowed to adapt the animal facility for 7 days prior to experimentation and had free access to food and water. All procedures used in this study were conducted in accordance with the Guide for the Care and Use of Laboratory Animals Center of Shenzhen University and approved by the Experimental Animal Ethics Committee of Shenzhen University (Permit No.2017011).

\section{Animal treatments and sample collection}

Mice were randomly divided into 7 groups according to the kinds and doses of QDs exposed, as follows: (1) hQDs high dose group (hQDs-H), treated with $25 \mathrm{mg} / \mathrm{kg}$ BW hQDs; (2) hQDs low dose group (hQDs-L), treated with $2.5 \mathrm{mg} / \mathrm{kg}$ BW hQDs; (3) aQDs high dose group (aQDs-H), treated with $25 \mathrm{mg} / \mathrm{kg}$ BW aQDs; (4) aQDs low dose group (aQDs-L), treated with $2.5 \mathrm{mg} / \mathrm{kg} \mathrm{BW}$ aQDs; (5) cQDs high dose group (cQDs$\mathrm{H}$ ), treated with $25 \mathrm{mg} / \mathrm{kg} \mathrm{BW}$ cQDs; (6) cQDs low dose group (cQDs-L), treated with $2.5 \mathrm{mg} / \mathrm{kg} \mathrm{BW} \mathrm{cQDs}$; (7) control group, treated with physiological saline. QDs were diluted in physiological saline and were injected intravenously into mice through the tail vein with a volume of $100 \mu \mathrm{L}$ per mouse. Mice in the control group were treated with the same volume of physiological saline. Observation of survival, food intake, fur, behavior, mental status, urine, feces and body weight were recorded daily for each mouse. At predetermined time points (1, 3, 7, 14 and 28 days), seven mice from each group were anesthetized using isoflurane. Blood was harvested from the posterior orbital venous plexus of mice. About $50 \mu \mathrm{L}$ of blood was collected with anticoagulant tubes for routine blood test. The rest of the blood was collected with procoagulant tube for biochemical analysis. Both routine blood test and biochemical analysis were operated immediately. Then the mice were sacrificed by cervical dislocation and the main organs (heart, liver, spleen, lung, kidney, and brain) were collected. Some pieces were fixed in tissue fixative (Wexis, China) for subsequent evaluation of histopathological changes. Some pieces were embedded in optimal cutting temperature compound (OCT, Sakura Finetek, USA) for tissue fluorescence imaging. Other fresh tissue samples were stored at $-80^{\circ} \mathrm{C}$ for measurements of In levels in organs. 


\section{Cryosection fluorescence microscopy}

Mice were administered intravenously with QDs with different surface functional groups at $25 \mathrm{mg} / \mathrm{kg} \mathrm{BW}$ or $2.5 \mathrm{mg} / \mathrm{kg} \mathrm{BW}$ and were sacrificed at various post-injection time points. The heart, liver, spleen, lung, kidney and brain was harvested, embedded into OCT compound and frozen at $-80^{\circ} \mathrm{C}$. The tissue frozen section was cut into $5 \mu \mathrm{m}$ thick by freezing microtome (CM3050S, Leica, Germany). Fluorescence microscopic imaging of QDs was observed by a fluorescence microscope (Axio Observer, ZEISS, Germany).

\section{Quantification of uptake in organs}

Liver, spleen and kidney of each mouse were digested in the microwave digestion

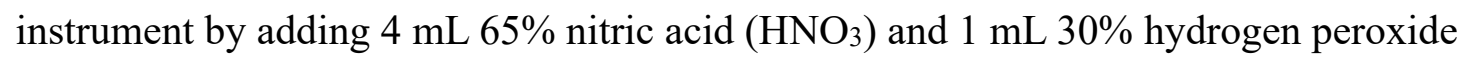
$\left(\mathrm{H}_{2} \mathrm{O}_{2}\right)$. The concentration of In in tissues was determined by ICP-MS (NexIon300X, PerkinElmer, USA). For all measurements, nitric acid blank and In standards was prepared and tested concurrently with test samples. Tissues from the control group at different post-injection time were dissolved in the similar manner. Concentration of In in the specific tissues was calculated using the following equations: [In] treated tissue $(\mu \mathrm{g} / \mathrm{g}$ wet tissue $)=[\mathrm{In}]$ tissue suspension/ wet weight of tissue.

\section{Histopathological examination}

Mice were sacrificed on Day1, Day 3, Day 7, Day 14 and Day 28 after the injection. Major organs including heart, liver, spleen, lung, kidney and brain were removed and fixed in tissue fixative. After gradient dehydration with different concentrations of alcohol in an automatic tissue dehydrator (APS300S, Leica, Germany), tissues were embedded in paraffin blocks by paraffin embedding station (Leica, Germany). Then the tissues were cut into $5 \mu \mathrm{m}$ thin slices by an ultra-thin semiautomatic microtome (RM2236, Leica, Germany) and adhered to the slides. After the slides were stained with hematoxylin and eosin (H\&E), histopathological morphology was evaluated under the microscope (Axio Observer, ZEISS, Germany) by an independent pathologist unaware of the treatment.

\section{Hematology analysis}


After the mice were anaesthetized, blood samples were harvested from the posterior orbital venous plexus of mice. About $50 \mu \mathrm{L}$ of blood were collected in tubes containing heparin sodium and routine blood analysis was determined by fully automatic five-classification hematology analyzer (BC5310, Mindray medical international limited, China). The specific hematology indexes in this study included white blood cell (WBC) count, neutrophils (Neu) percentage, lymphocyte (Lym) percentage, red blood cell (RBC) count, hematocrit (HCT), mean corpuscular volume (MCV), coefficient of variation of RBC volume distributing width (RDW-CV), hemoglobin concentration (HGB), mean corpuscular hemoglobin (MCH), platelet (PLT) count, thromboplastin (PCT), and mean platelet volume (MPV).

\section{Serum biochemical analysis}

The whole blood of mice was collected in disposable venous blood vessels containing separating gel and the serum was obtained by centrifugation for 15 mins at $4^{\circ} \mathrm{C}, 3500 \mathrm{rpm} / \mathrm{min}$. The serum biochemical parameters were detected by automatic blood biochemical analyzer (BS-220, Mindray medical international limited, China). All matching reagents were purchased from Mindray medical international limited, China. The specific biochemical indexes in this study included alanine aminotransferase (ALT), aspartate transaminase (AST), alkaline phosphatase (ALP), total protein (TP), albumin (ALB), globulin (GLB), the albumin and globulin ratio $(\mathrm{A} / \mathrm{G})$, total bilirubin (T-Bil), direct bilirubin (D-Bil), triglyceride (TG), total cholesterol (TC), high density lipoprotein (HDL-C), low-density lipoprotein (LDL-C), glucose (GLU), lactate dehydrogenase (LDH), creatine kinase (CK), creatine kinase isoenzyme (CK-MB), $\alpha$-hydroxy butyrate dehydrogenase ( $\alpha$-HBDH), uric acid (UA), urea and creatinine (CREA).

\section{Statistical analysis}

All statistical analysis was performed by SPSS 22.0 statistical software packages and figures were drawn with GraphPad Prism software package. Data was expressed as mean \pm standard deviation (SD). The difference among the different groups was compared by one-way ANOVA. Results were considered significant if $P<0.05$. 


\section{List of abbreviations}

QDs: quantum dots; InP: Indium phosphide; PL: photoluminescence; NIR: near infrared; BW: body weight; In: Indium; hQDs: hydroxylated QDs; aQDs, amino; cQDs: carboxylic QDs; TEM: transmission electron microscopy; OCT: optimal cutting temperature compound; WBC: white blood cell; Neu: neutrophils; Lym: lymphocyte; RBC: red blood cell; HCT: hematocrit; MCV: mean corpuscular volume; RDW-CV: coefficient of variation of RBC volume distributing width; HGB: hemoglobin concentration; $\mathrm{MCH}$ : mean corpuscular hemoglobin; PLT: platelet; PCT: thromboplastin; MPV: mean platelet volume; ALT: alanine aminotransferase; AST: aspartate transaminase; ALP: alkaline phosphatase; TP: total protein; ALB: albumin; GLB: globulin; A/G: the albumin and globulin ratio; T-Bil: total bilirubin; D-Bil: direct bilirubin; TG: triglyceride; TC: total cholesterol; HDL-C: high density lipoprotein; LDL-C: low-density lipoprotein; GLU: glucose; LDH: lactate dehydrogenase; CK: creatine kinase; CK-MB: creatine kinase isoenzyme; $\alpha$-HBDH: $\alpha$-hydroxy butyrate dehydrogenase; UA: uric acid; CREA: creatinine; SD: standard deviation; ICP-MS: inductively coupled plasma mass spectrometry.

\section{Availability of data and materials}

All data generated or analyzed during this study are included in this published article.

\section{Acknowledgements}

Thanks to Instrumental Analysis Center of Shenzhen University.

\section{Authors' contributions}

LL, YC and DL performed the experiments, and with LL, GL designed the experiments and drafted the manuscript. XW, GX, WJ and GL contributed to the data analysis, manuscript preparation, and manuscript review and revision process. XY, TC and DX participated in the animal experiments. All authors read and approved the final manuscript. 


\section{Funding}

The work is supported by grants from National Natural Science Foundation of China (NSFC) (No. 21677102, 61935012, 31671491 and 81772002), the Chinese Ministry of Science and Technology (No. 2016YFC0904600), Shenzhen Science and Technology project (No. JCYJ20170817093725277, No. GJHZ20170313111237888, No. JCYJ20170817102634964) and No. JCYJ20170818143334365), Natural Science Foundation of SZU (No. 827-000100), and SZU medical young scientists program.

\section{Ethics approval and consent to participate}

The study was conducted in accordance with the Guide for the Care and Use of Laboratory Animals Center of Shenzhen University and approved by the Experimental Animal Ethics Committee of Shenzhen University (Permit No.2017011).

\section{Declaration of interest statement}

No conflict of interest in the submission of this manuscript is declared.

\section{Consent for publication}

All the authors have approved the manuscript and agree with submission to your esteemed journal.

\section{Author details}

${ }^{1}$ Base for International Science and Technology Cooperation: Carson Cancer Stem Cell Vaccines R\&D Center, Shenzhen Key Lab of Synthetic Biology, Department of Physiology, School of Basic Medical Sciences, Shenzhen University, Shenzhen 518055, China

2 Key Laboratory of Optoelectronics Devices and Systems of Ministry of Education/Guangdong Province, College of Optoelectronic Engineering, Shenzhen University, Shenzhen 518060, China

${ }^{3}$ Guangdong Key Laboratory for Biomedical Measurements and Ultrasound Imaging, School of Biomedical Engineering, Health Science Center, Shenzhen University, 
Shenzhen, 518055, China

\section{References}

[1] W.C.W. Chan, D.J. Maxwell, X.H. Gao, R.E. Bailey, M.Y. Han, S.M. Nie. Luminescent quantum dots for multiplexed biological detection and imaging. Curr Opin Biotech. 2002; 13(1): 40-46.

[2] S. Zarco-Fernandez, A.M. Coto-Garcia, R. Munoz-Olivas, J. Sanz-Landaluze, S. Rainieri, C. Camara. Bioconcentration of ionic cadmium and cadmium selenide quantum dots in zebrafish larvae. Chemosphere. 2016; 148: 328-35.

[3] L. Li, D.H. Yin, K. Xu, Y.S. Liu, D.D. Song, J. Wang, C. Zhao, X.L. Song, J. Li. A sandwich immunoassay for brucellosis diagnosis based on immune magnetic beads and quantum dots. J Pharmaceut Biomed. 2017; 141: 79-86.

[4] G.M. Lin, L. Li, N. Panwar, J. Wang, S.C. Tjin, X.M. Wang, K.T. Yong. Non-viral gene therapy using multifunctional nanoparticles: Status, challenges, and opportunities. Coordin Chem Rev. 2018; 374: 133-52.

[5] R. Yadav, P. Madhukar Chaudhary, B. Subramani, S. Toraskar, H. Bavireddi, R.V. Murthy, S. Sangabathuni, R. Kikkeri. Imaging and Targeting of the alpha(2-6) and alpha(2-3) Linked Sialic Acid Quantum Dots in Zebrafish and Mouse Models. ACS Appl Mater Interfaces. 2018; 10(34): 28322-30.

[6] D. Li, B. Kristal, Y.J. Wang, J.W. Feng, Z.G. Lu, G. Yu, Z. Chen, Y.Z. Li, X.G. Li, X.G. Xu. Enhanced Efficiency of InP-Based Red Quantum Dot Light-Emitting Diodes. Acs Applied Materials \& Interfaces. 2019; 11(37): 34067-75.

[7] Z. Ranjbar-Navazi, Y. Omidi, M. Eskandani, S. Davaran. Cadmium-free quantum dot-based theranostics. Trac-Trends in Analytical Chemistry. 2019; 118: 386400 .

[8] L. Zhang, X.Q. Yang, J. An, S.D. Zhao, T.Y. Zhao, F. Tan, Y.C. Cao, Y.D. Zhao. In vivo tumor active cancer targeting and CT-fluorescence dual-modal imaging with nanoprobe based on gold nanorods and $\mathrm{InP} / \mathrm{ZnS}$ quantum dots. J Mater Chem B. 2018; 6(17): 2574-83.

[9] D.J. Bharali, D.W. Lucey, H. Jayakumar, H.E. Pudavar, P.N. Prasad. Folatereceptor-mediated delivery of InP quantum dots for bioimaging using confocal and two-photon microscopy. J Am Chem Soc. 2005; 127(32): 11364-71.

[10] K.T. Yong, H. Ding, I. Roy, W.C. Law, E.J. Bergey, A. Maitra, P.N. Prasad. Imaging Pancreatic Cancer Using Bioconjugated InP Quantum Dots. Acs Nano. 2009; 3(3): 502-10.

[11] E. Yaghini, H. Turner, A. Pilling, I. Naasani, A.J. MacRobert. In vivo biodistribution and toxicology studies of cadmium-free indium-based quantum dot nanoparticles in a rat model. Nanomed-Nanotechnol. 2018; 14(8): 2644-55.

[12] V. Brunetti, H. Chibli, R. Fiammengo, A. Galeone, M.A. Malvindi, G. Vecchio, R. Cingolani, J.L. Nadeau, P.P. Pompa. InP/ZnS as a safer alternative to CdSe/ZnS core/shell quantum dots: in vitro and in vivo toxicity assessment. Nanoscale. 
2013; 5(1): 307-17.

[13] K. Yamazaki, A. Tanaka, M. Hirata, M. Omura, Y. Makita, N. Inoue, K. Sugio, K. Sugimachi. Long term pulmonary toxicity of indium arsenide and indium phosphide instilled intratracheally in hamsters. J Occup Health. 2000; 42(4): 169-78.

[14] Y. Chen, Y. Yang, F. Ou, L. Liu, X.H. Liu, Z.J. Wang, L. Jin. InP/ZnS QDs exposure induces developmental toxicity in rare minnow (Gobiocypris rarus) embryos. Environmental Toxicology and Pharmacology. 2018; 60: 28-36.

[15] L. Yu, X. Tian, D. Gao, Y. Lang, X.X. Zhang, C. Yang, M.M. Gu, J. Shi, P.K. Zhou, Z.F. Shang. Oral administration of hydroxylated-graphene quantum dots induces intestinal injury accompanying the loss of intestinal stem cells and proliferative progenitor cells. Nanotoxicology. 2019; 1-13.

[16] N. Murase, M. Horie, T. Sawai, K. Kawasaki. Silica layer-dependent leakage of cadmium from $\mathrm{CdSe} / \mathrm{ZnS}$ quantum dots and comparison of cytotoxicity with polymer-coated analogues. J Nanopart Res. 2019; 21(1): 10.

[17] H. Zheng, L.J. Mortensen, S. Ravichandran, K. Bentley, L.A. DeLouise. Effect of Nanoparticle Surface Coating on Cell Toxicity and Mitochondria Uptake. J Biomed Nanotechnol. 2017; 13(2): 155-66.

[18] J. Liu, R. Hu, J.W. Liu, B.T. Zhang, Y.C. Wang, X. Liu, W.C. Law, L.W. Liu, L. Ye, K.T. Yong. Cytotoxicity assessment of functionalized CdSe, CdTe and InP quantum dots in two human cancer cell models. Mat Sci Eng C-Mater. 2015; 57: 222-31.

[19] Y. Du, Y.Y. Zhong, J.J. Dong, C.T. Qian, S.A. Sun, L.P. Gao, D.Z. Yang. The effect of PEG functionalization on the in vivo behavior and toxicity of CdTe quantum dots. Rsc Adv. 2019; 9(22): 12218-25.

[20] J.J. Song, Z. Dai, W.J. Guo, Y. Li, W.T. Wang, N.N. Li, J.F. Wei. Preparation of $\mathrm{CdTe} / \mathrm{CdS} / \mathrm{SiO} 2$ Core/Multishell Structured Composite Nanoparticles. J Nanosci Nanotechno. 2013; 13(10): 6924-27.

[21] X.Z. Tang, J. Sun, T. Ge, K.Q. Zhang, Q.Y. Gui, S.T. Zhang, W.D. Chen. PEGylated liposomes as delivery systems for Gambogenic acid: Characterization and in vitro/in vivo evaluation. Colloid Surface B. 2018; 172: 26-36.

[22] T. Chen, L. Li, G.X. Xu, X.M. Wang, J. Wang, Y.J. Chen, W.X. Jiang, Z.W. Yang, G.M. Lin. Cytotoxicity of InP/ZnS Quantum Dots With Different Surface Functional Groups Toward Two Lung-Derived Cell Lines. Front Pharmacol. 2018; 9: 763.

[23] M. Allocca, L. Mattera, A. Bauduin, B. Miedziak, M. Moros, L. De Trizio, A. Tino, P. Reiss, A. Ambrosone, C. Tortiglione. An Integrated Multilevel Analysis Profiling Biosafety and Toxicity Induced by Indium- and Cadmium-Based Quantum Dots in Vivo. Environmental Science \& Technology. 2019; 53(7): 3938-47.

[24] Y. Chen, Y. Yang, F. Ou, L. Liu, X.H. Liu, Z.J. Wang, L. Jin. InP/ZnS QDs exposure induces developmental toxicity in rare minnow (Gobiocypris rarus) embryos. Environ Toxicol Pharmacol. 2018; 60: 28-36. 
[25] Y.C. Xie, B. Wan, Y. Yang, X.J. Cui, Y. Xin, L.H. Guo. Cytotoxicity and autophagy induction by graphene quantum dots with different functional groups. J Environ Sci-China. 2019; 77: 198-209.

[26] M.M. Haque, H.Y. Im, J.E. Seo, M. Hasan, K. Woo, O.S. Kwon. Acute toxicity and tissue distribution of $\mathrm{CdSe} / \mathrm{CdS}-\mathrm{MPA}$ quantum dots after repeated intraperitoneal injection to mice. J Appl Toxicol. 2013; 33(9): 940-50.

[27] W.G. Kreyling, A.M. Abdelmonem, Z. Ali, F. Alves, M. Geiser, N. Haberl, R. Hartmann, S. Hirn, D.J. de Aberasturi, K. Kantner, G. Khadem-Saba, J.M. Montenegro, J. Rejman, T. Rojo, I.R. de Larramendi, R. Ufartes, A. Wenk, W.J. Parak. In vivo integrity of polymer-coated gold nanoparticles. Nat Nanotechnol. 2015; 10(7): 619-23.

[28] D. Docter, D. Westmeier, M. Markiewicz, S. Stolte, S.K. Knauer, R.H. Stauber. The nanoparticle biomolecule corona: lessons learned - challenge accepted? Chem Soc Rev. 2015; 44(17): 6094-121.

[29] N. Liu, Y. Mu, Y. Chen, H.B. Sun, S.H. Han, M.M. Wang, H. Wang, Y.B. Li, Q. $\mathrm{Xu}$, P.L. Huang, Z.W. Sun. Degradation of aqueous synthesized CdTe/ZnS quantum dots in mice: differential blood kinetics and biodistribution of cadmium and tellurium. Particle and Fibre Toxicology. 2013; 10: 37.

[30] H.D. Duong, S. Yang, Y.W. Seo, J.I. Rhee. Effects of CdSe and CdSe/ZnS Core/Shell Quantum Dots on Singlet Oxygen Production and Cell Toxicity. J Nanosci Nanotechno. 2018; 18(3): 1568-76.

[31] G.Z. Wu, X. Chen, X.Y. Ma, L. Liu. Toxicity evaluation of CdSe nanorods on macrophages and the protective effect of resveratrol. J Nanopart Res. 2019; 21(2): 38.

[32] Y.H. Lu, S.C. Xu, H.Y. Chen, M.D. He, Y.C. Deng, Z.W. Cao, H.F. Pi, C.H. Chen, M. Li, Q.L. Ma, P. Gao, Y. Ji, L. Zhang, Z.P. Yu, Z. Zhou. CdSe/ZnS quantum dots induce hepatocyte pyroptosis and liver inflammation via NLRP3 inflammasome activation. Biomaterials. 2016; 90: 27-39.

[33] J.R. Roberts, J.M. Antonini, D.W. Porter, R.S. Chapman, J.F. Scabilloni, S.H. Young, D. Schwegler-Berry, V. Castranova, R.R. Mercer. Lung toxicity and biodistribution of $\mathrm{Cd} / \mathrm{Se}-\mathrm{ZnS}$ quantum dots with different surface functional groups after pulmonary exposure in rats. Particle and Fibre Toxicology. 2013; 10: 5 .

[34] D. Mo, L. Hu, G.M. Zeng, G.Q. Chen, J. Wan, Z.G. Yu, Z.Z. Huang, K. He, C. Zhang, M. Cheng. Cadmium-containing quantum dots: properties, applications, and toxicity. Appl Microbiol Biot. 2017; 101(7): 2713-33.

[35] S.J. Soenen, J.M. Montenegro, A.M. Abdelmonem, B.B. Manshian, S.H. Doak, W.J. Parak, S.C. De Smedt, K. Braeckmans. The effect of nanoparticle degradation on poly(methacrylic acid)-coated quantum dot toxicity: The importance of particle functionality assessment in toxicology. Acta Biomater. 2014; 10(2): 732-41.

[36] G. Veronesi, M. MorosIl, H. Castillo-Michel, L. Mattera, G. Onorato, K.D. Wegner, W.L. Ling, P. Reiss, C. Tortiglione. In Vivo Biotransformations of Indium Phosphide Quantum Dots Revealed by X-Ray Microspectroscopy. Acs Applied 
Materials \& Interfaces. 2019; 11(39): 35630-40.

[37] H.S. Choi, W. Liu, P. Misra, E. Tanaka, J.P. Zimmer, B.I. Ipe, M.G. Bawendi, J.V. Frangioni. Renal clearance of quantum dots. Nature Biotechnology. 2007; 25(10): 1165-70.

[38] M.G. Lu, K.T. Al-Jamal, K. Kostarelos, J. Reineke. Physiologically Based Pharmacokinetic Modeling of Nanoparticles. Acs Nano. 2010; 4(11): 6303-17

[39] J.A.J. Fitzpatrick, S.K. Andreko, L.A. Ernst, A.S. Waggoner, B. Ballou, M.P. Bruchez. Long-term Persistence and Spectral Blue Shifting of Quantum Dots in Vivo. Nano Letters. 2009; 9(7): 2736-41.

[40] K. Wu, D.Q. Su, J.M. Liu, R. Saha, J.P. Wang. Magnetic nanoparticles in nanomedicine: a review of recent advances. Nanotechnology. 2019; 30(50): 502003.

[41] Y. Tang, S. Han, H. Liu, X. Chen, L. Huang, X. Li, J. Zhang. The role of surface chemistry in determining in vivo biodistribution and toxicity of $\mathrm{CdSe} / \mathrm{ZnS}$ coreshell quantum dots. Biomaterials. 2013; 34(34): 8741-55. 
Figures

A
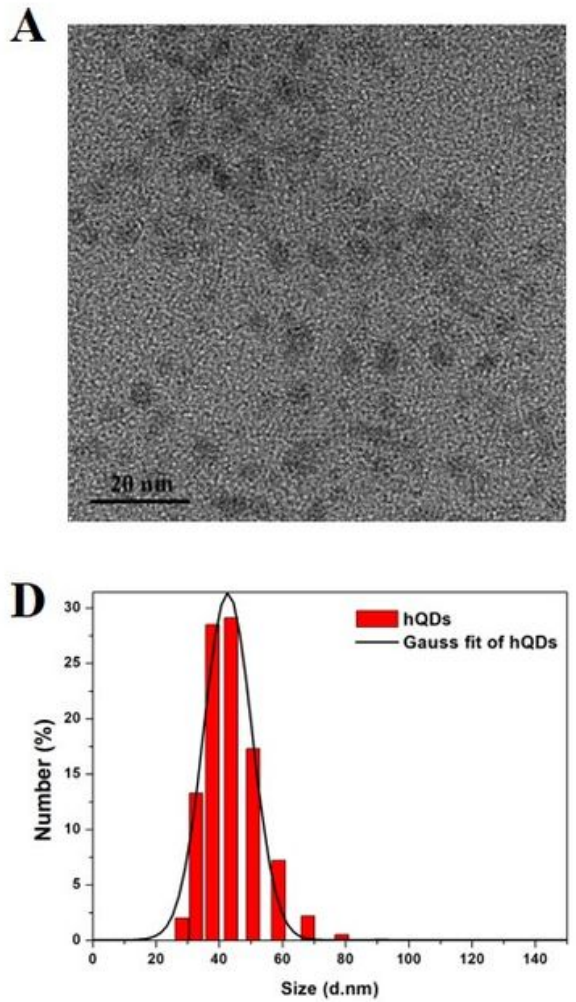

G

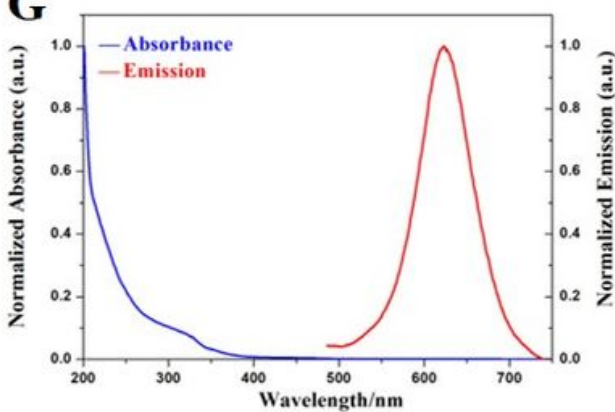

B

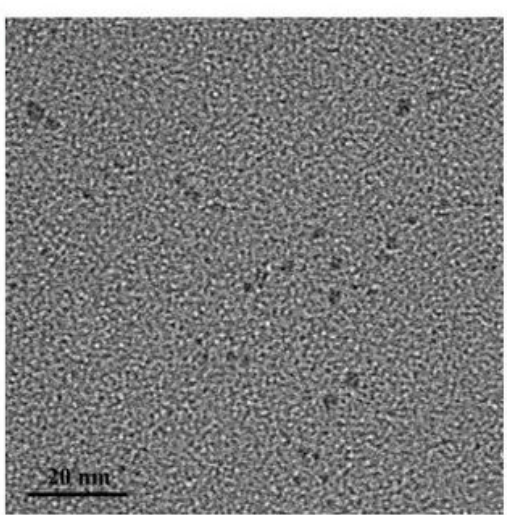

E

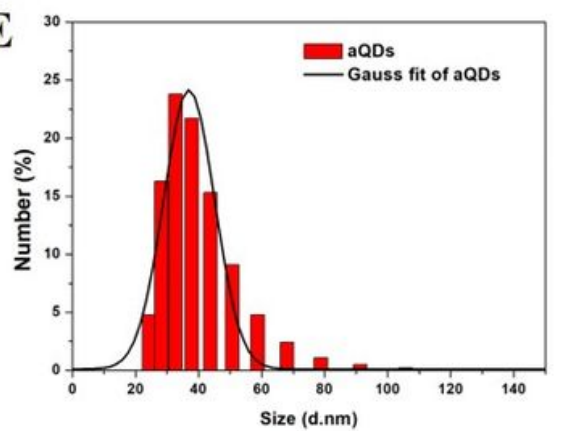

H

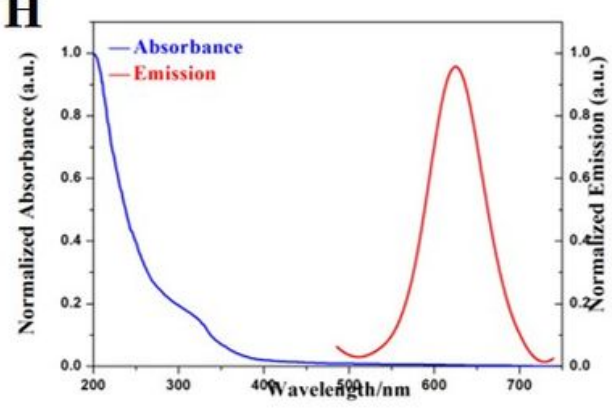

C

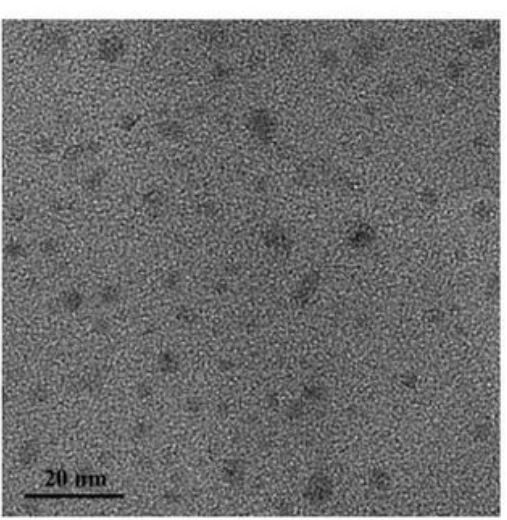

F

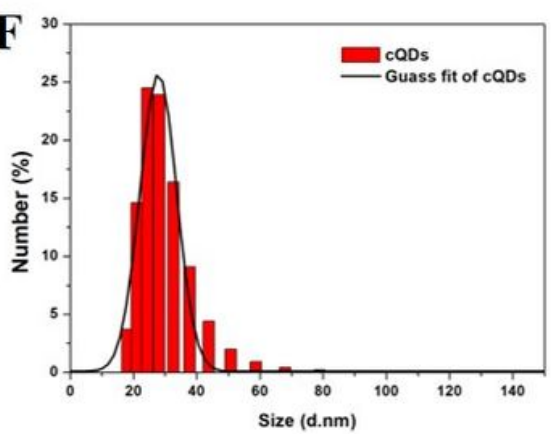

I

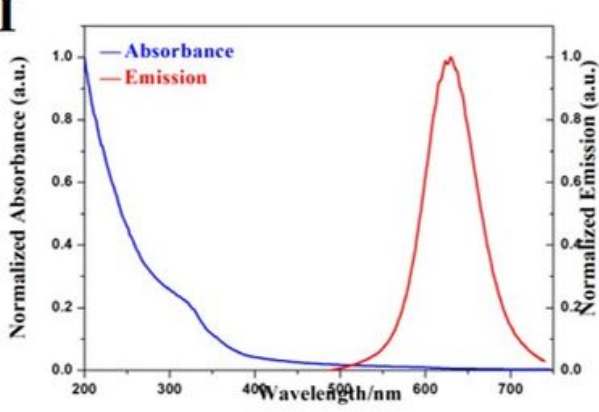

Figure 1

Characterization of InP/ZnS QDs with different surface modifications. (A-C) TEM image of hQDs, aQDs, and CQDs, respectively. (D-F) DLS of hQDs, aQDs and CQDs dispersed in deionized water. (G-I) Normalized absorption spectra and normalized PL spectra of hQDs, aQDs and CQDs, respectively 

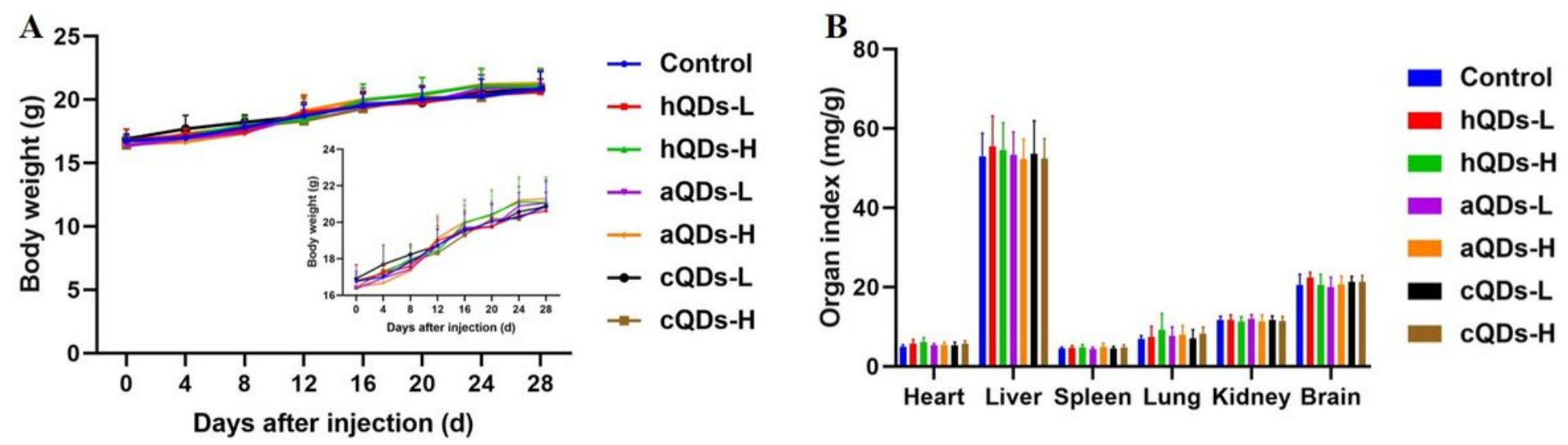

Figure 2

Body weight and organ index in mice. (A) The body weight curve of mice continuously monitored for 28 days (Inset showed the magnified state of the body weight curve). (B) The main organ index of mice on Day 28 after administration.

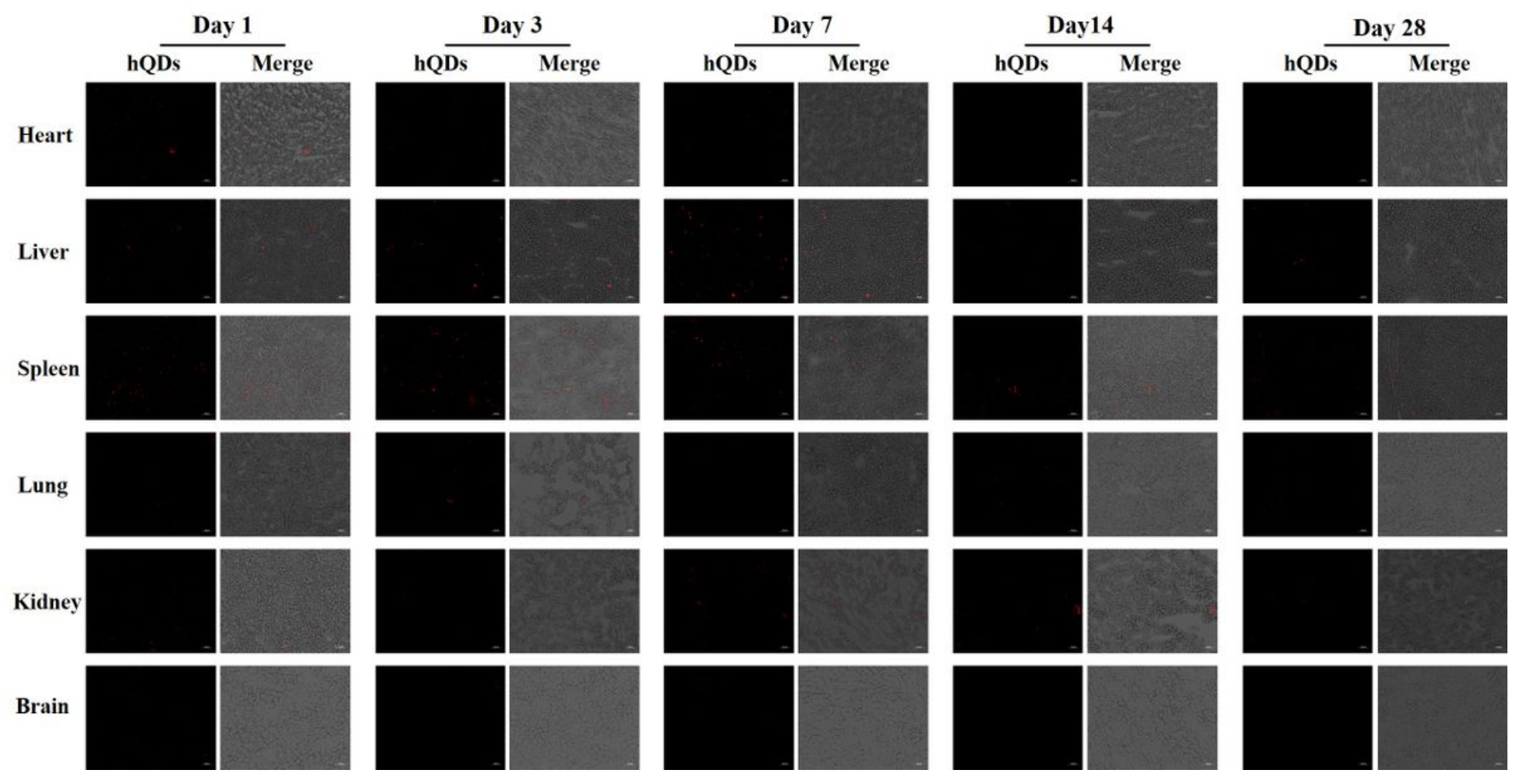

Figure 3

Fluorescence images of main organ tissues of mice on Day 1, Day 3, Day 7, Day 14 and Day 28 after treated with $25 \mathrm{mg} / \mathrm{kg} \mathrm{BW}$ hQDs (scale bar: $50 \mu \mathrm{m}$ ). 


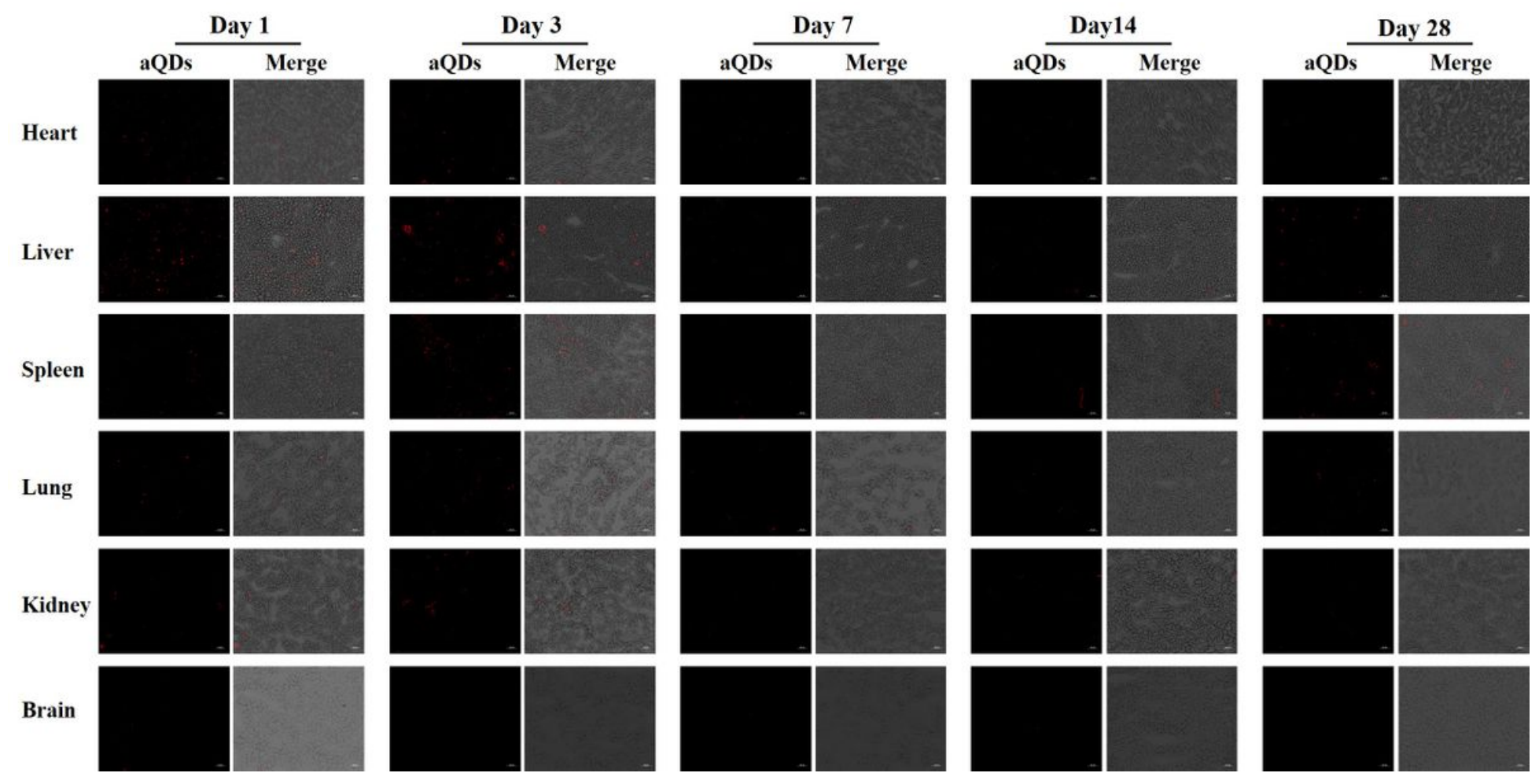

Figure 4

Fluorescence images of main organ tissues of mice on Day1, Day 3, Day 7, Day 14 and Day 28 after treated with $25 \mathrm{mg} / \mathrm{kg} \mathrm{BW}$ aQDs (scale bar: $50 \mu \mathrm{m}$ ).

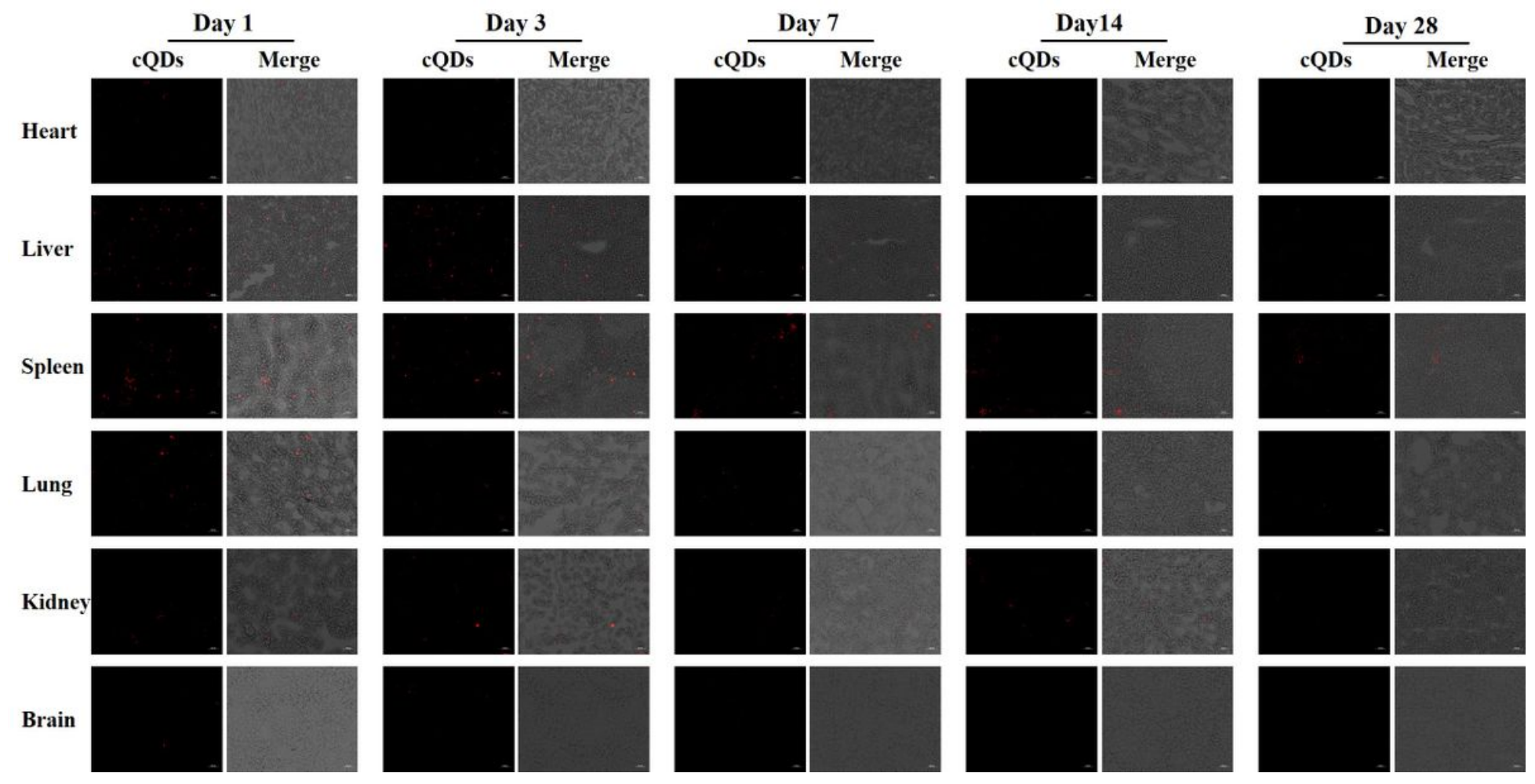

Figure 5 
Fluorescence images of main organ tissues of mice on Day1, Day 3, Day 7, Day 14 and Day 28 after treated with $25 \mathrm{mg} / \mathrm{kg} \mathrm{BW}$ cQDs (scale bar: $50 \mu \mathrm{m}$ ).
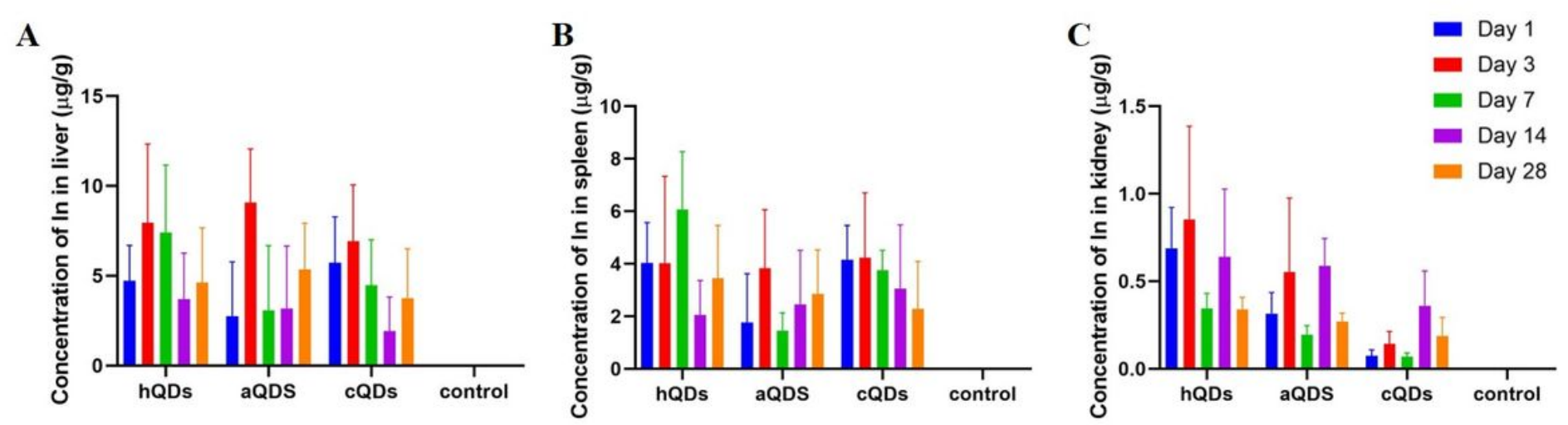

\section{Figure 6}

The In element concentration in organs over a period of 28 days in mice following administration of three InP/ZnS QDs at 25 mg/kg BW. (A) Liver tissues. (B) Spleen tissues. (C) Kidney tissues.

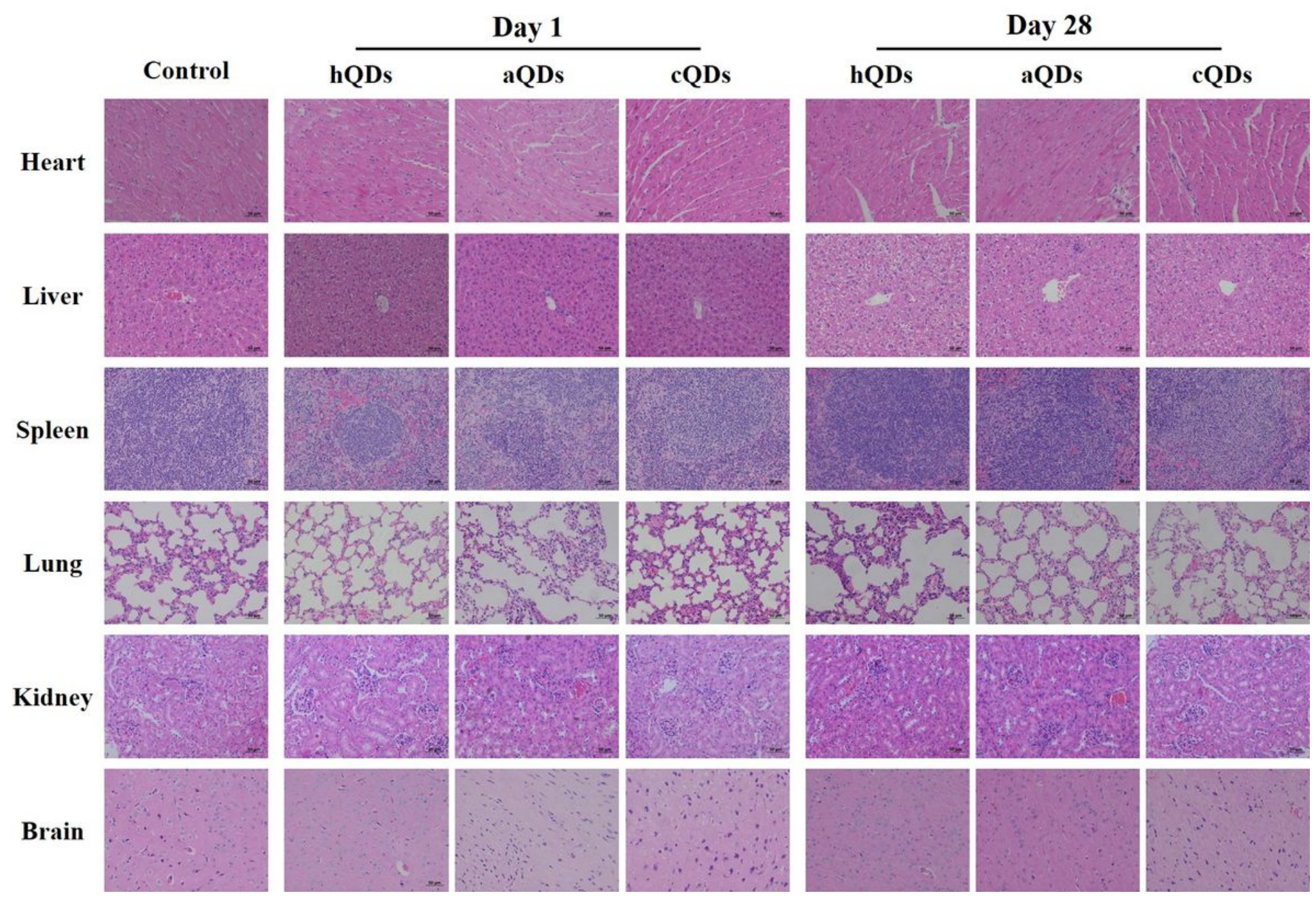

Figure 7 
Representative histological images of major organs including heart, liver, spleen, lung, kidney and brain collected from the control group mice and different surface functionalized QDs treated mice following intravenous injection at dose of $25 \mathrm{mg} / \mathrm{kg}$ at 1 day and 28 days post-injection (scale bar: $50 \mu \mathrm{m}$ ).

\section{Control $\square$ hQDs-L $\square$ hQDs-H $\square$ aQDs-L $\square$ aQDs-H $\square$ cQDs-L $\square$ cQDs-H}
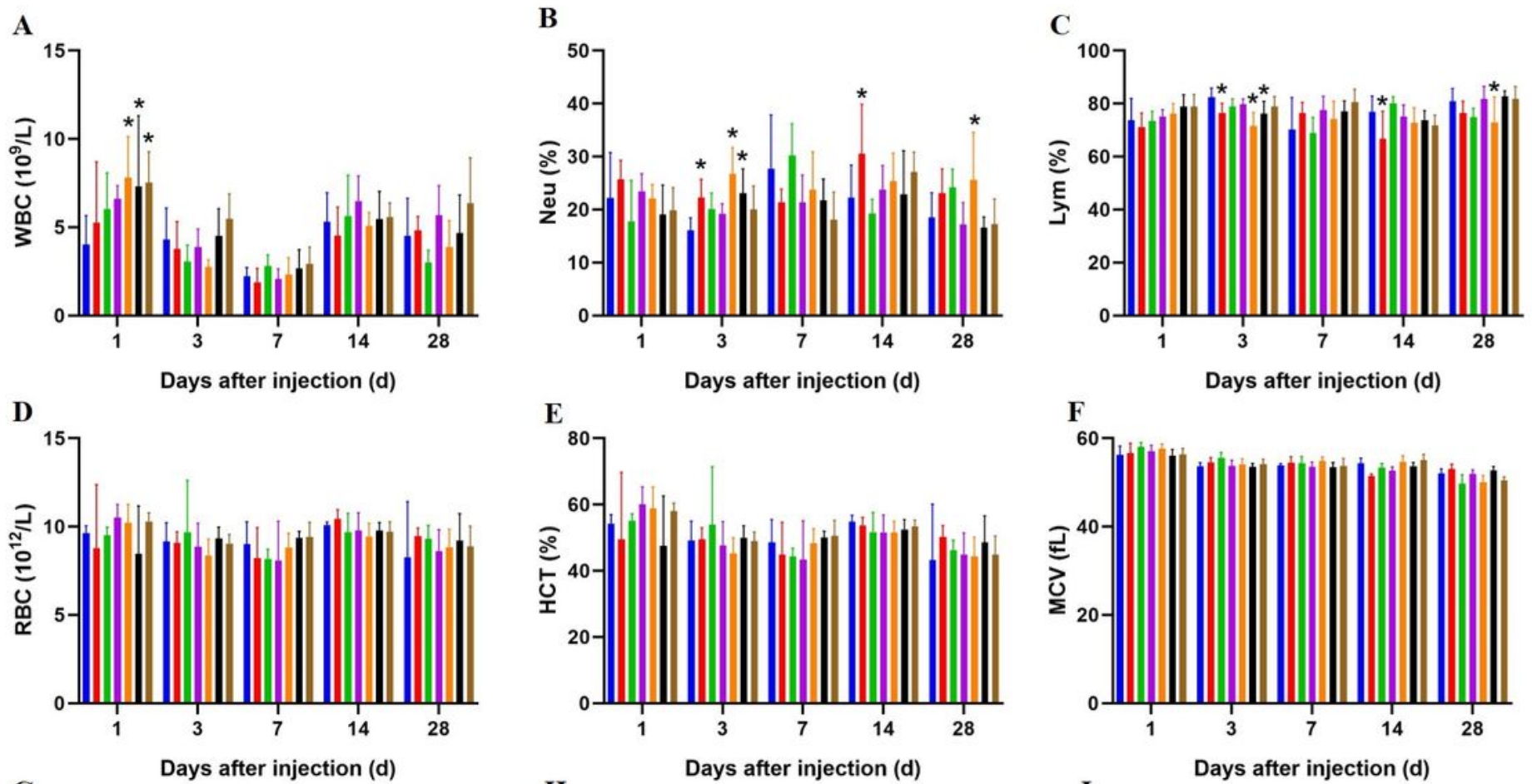

$\mathbf{E}$

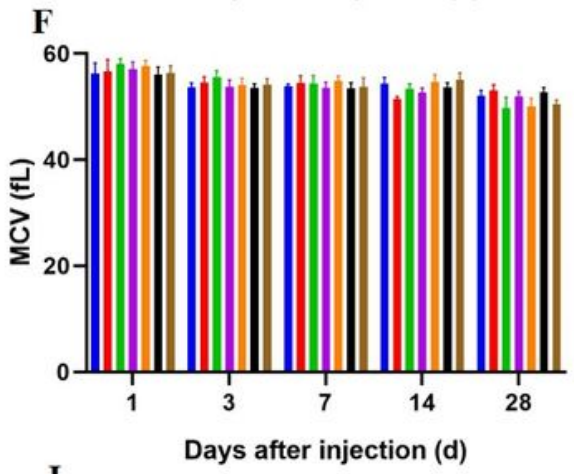

G
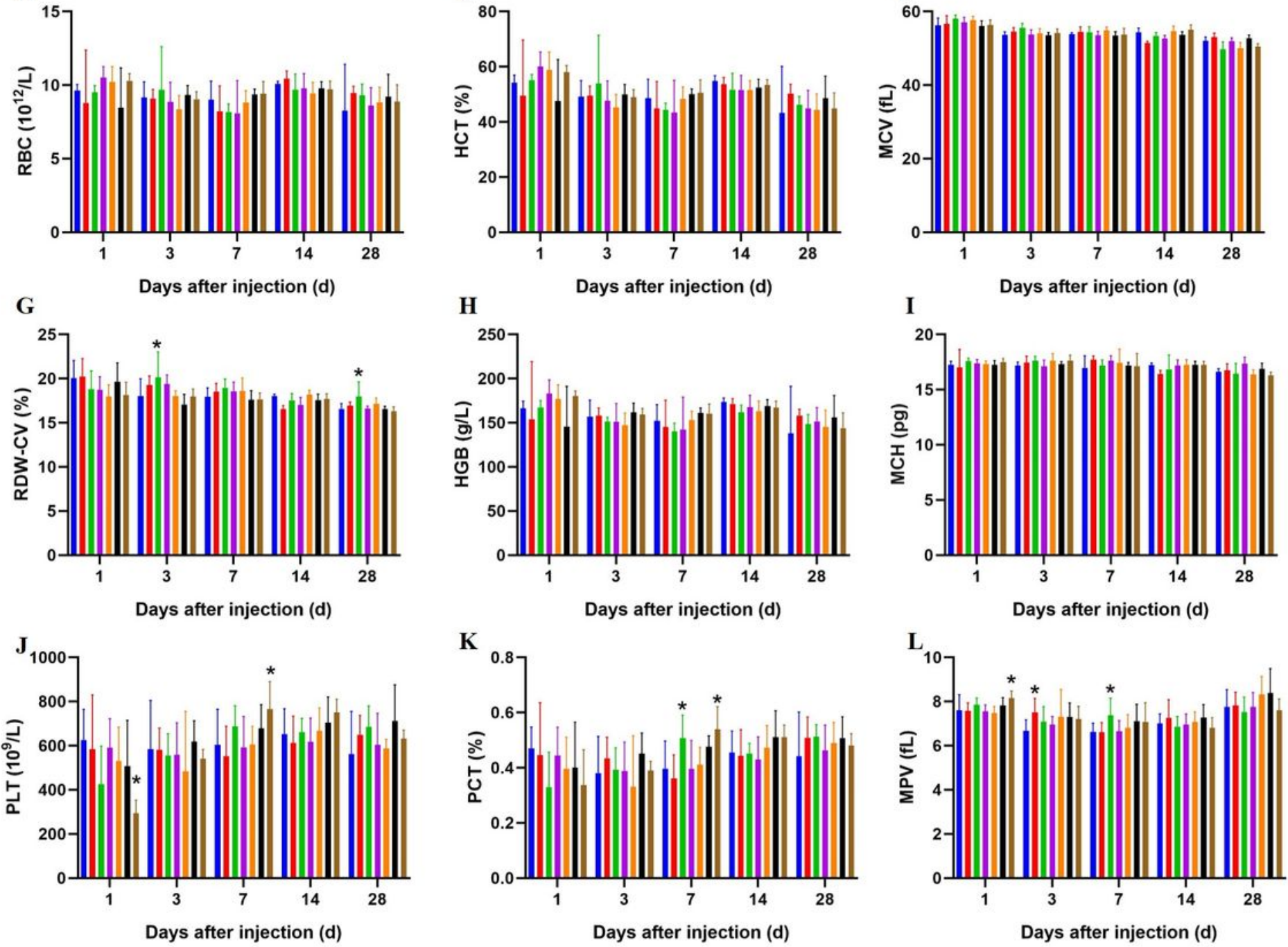

Figure 8

Hematology results of the control group mice and different surface functionalized QDs treated mice following intravenous injection at 1, 3, 7, 14 and 28 days post-injection. (A) White blood cell (WBC). (B) 
Neutrophils (Neu) percentage. (C) Lymphocyte (Lym) percentage. (D) Red blood cell (RBC) count. (E) Hematocrit(HCT) levels. (F) Mean corpuscular volume (MCV) levels. (G) Coefficient of variation of RBC volume distributing width (RDW-CV) levels. (H) Hemoglobin concentration (HGB) levels. (I) Mean corpuscular hemoglobin (MCH) levels. (J) Platelet (PLT) count. (K) Thromboplastin (PCT) levels. (L) Mean platelet volume (MPV) levels. ( ${ }^{*}$ Significantly different compared to control group at the same sampling time, $\mathrm{P}<0.05$.)
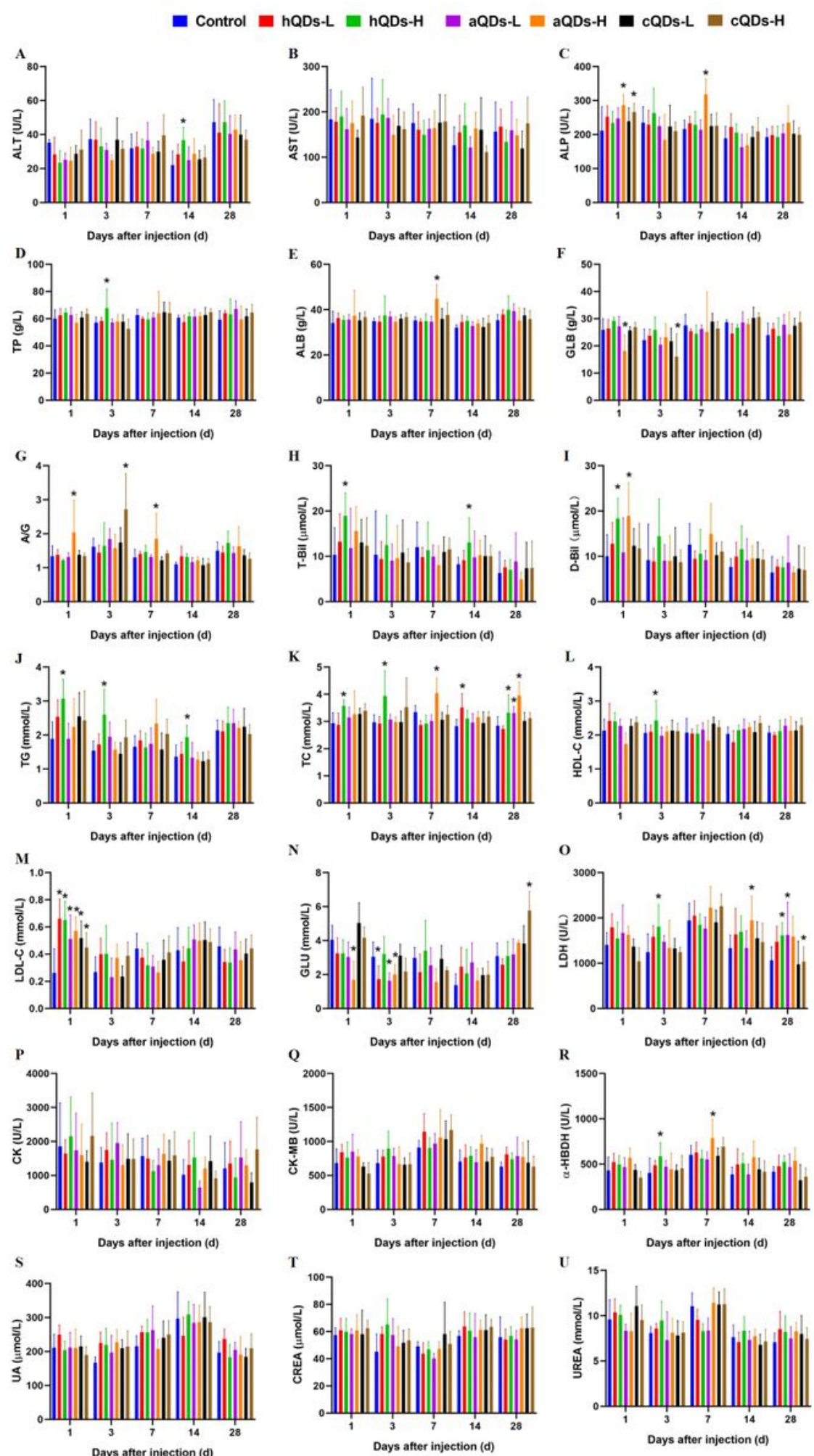

Figure 9 
Serum biochemical results of the control group mice and different surface functionalized QDs treated mice following intravenous injection at 1, 3, 7, 14 and 28 days post-injection. (A) Alanine aminotransferase (ALT) levels. (B) Aspartate transaminase (AST) levels. (C) Alkaline phosphatase (ALP) levels. (D) Total protein (TP) levels. (E) Albumin (ALB) levels. (F) Globulin (GLB) levels. (G) Albumin and globulin ratio (A/G). (H) Total bilirubin (T-Bil) levels. (I) Direct bilirubin (D-Bil) levels. (J) Triglyceride (TG) levels. (K) Total cholesterol (TC) levels. (L) High density lipoprotein (HDL-C) levels. (M) Low-density lipoprotein (LDL-C) levels. (N) Glucose (GLU) levels. (O) Lactate dehydrogenase (LDH) levels. (P) Creatine kinase (CK) levels. (Q) Creatine kinase isoenzyme (CK-MB) levels. (R) a-Hydroxybutyrate dehydrogenase (a-HBDH) levels. (S) Uric acid (UA) levels. (T) Urea levels. (U) Creatinine (CREA) levels. (`Significantly different compared to control group at the same sampling time, $\mathrm{P}<0.05$.)

\section{Supplementary Files}

This is a list of supplementary files associated with this preprint. Click to download.

- table.pdf 